Pacific

Journal of

Mathematics

\title{
TRIANGULATIONS OF FIBRE-FREE HAKEN 3-MANIFOLDS
}

AleKsandar MiJatović 


\title{
TRIANGULATIONS OF FIBRE-FREE HAKEN 3-MANIFOLDS
}

\author{
ALEKSANDAR MiJATOVIĆ
}

\begin{abstract}
It is not known whether there exists a computable function bounding the number of Pachner moves needed to connect any two triangulations of a compact 3-manifold. In this paper we find an explicit bound of this kind for all Haken 3-manifolds that contain no fibred submanifolds as strongly simple pieces of their JSJ-decomposition. The explicit formula for the bound is in terms of the number of tetrahedra in the two triangulations. This implies a conceptually trivial algorithm for recognising any nonfibred knot complement among all 3-manifolds.
\end{abstract}

\section{Introduction}

It is a nontrivial fact, proved in [Pachner 1991], that any triangulation of a compact PL $n$-manifold can be transformed into any other triangulation of the same manifold by a finite sequence of simplicial moves and simplicial isomorphisms. The moves can be described as follows.

Definition. Let $T$ be a triangulation of a compact PL $n$-manifold $M$. Suppose $D$ is a combinatorial $n$-disc which is a subcomplex both of $T$ and of the boundary of a standard $(n+1)$-simplex $\Delta^{n+1}$. A Pachner move consists of changing $T$ by removing the subcomplex $D$ and inserting $\partial \Delta^{n+1}-\operatorname{int} D$ (see Figure 1 for the case $n=3)$.
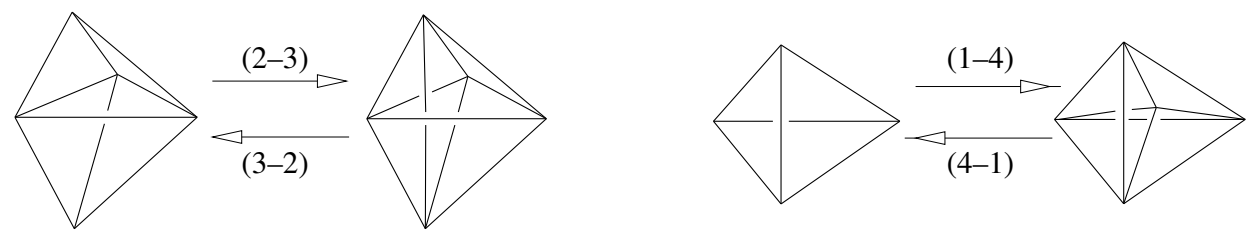

Figure 1. Three-dimensional Pachner moves.

It is an immediate consequence of the definition that precisely $n+1$ Pachner moves are possible in dimension $n$. If our $n$-manifold $M$ has nonempty boundary,

Keywords: Haken 3-manifold, triangulation, hierarchy, Pachner moves. 
the moves from this definition do not alter the induced triangulation of $\partial M$. But changing the simplicial structure (we use the term synonymously with "triangulation") of the boundary with an (n-1)-dimensional Pachner move can be achieved by gluing onto (or removing from) our manifold $M$ the standard $n$-simplex $\Delta^{n}$ that exists by the definition of the move. Our aim in this paper is to consider the triangulations of Haken 3-manifolds. Their JSJ-decompositions (see Section 2 for the precise definition) consist of strongly simple pieces, $I$-bundles and Seifert fibred spaces. The strongly simple pieces are the ones that contain all the interesting topological information about the manifold and also have the crucial property of being atoroidal (meaning that all incompressible tori in them are boundary parallel) and are hence hyperbolic. It is precisely the strongly simple submanifolds that we have to make additional hypothesis on in the next theorem.

Convention. We let $E$ denote the base-2 exponential function $x \mapsto 2^{x}$, and write $E^{k}$ for the $k$-fold composition of $E$ with itself.

Theorem 1.1. Let $M$ be a Haken 3-manifold whose JSJ-decomposition does not contain strongly simple pieces which are surface bundles or semibundles. Let $P$ and $Q$ be two triangulations of $M$ containing $p$ and $q$ tetrahedra respectively. Then there exists a sequence of Pachner moves, of length at most

$$
E^{2^{a p}}(p)+E^{2^{a q}}(q),
$$

that transforms $P$ into a triangulation isomorphic to $Q$. The constant a is bounded above by 200. The homeomorphism of $M$ realizing this simplicial isomorphism is supported in the characteristic submanifold of $M$ and does not permute the components of $\partial M$.

The triangulations appearing in the theorem are allowed to be noncombinatorial, meaning that the simplices are not (necessarily) uniquely determined by their vertices. Since the formula in Theorem 1.1 is explicit, it gives a conceptually trivial algorithm (see Proposition 1.3 in [Mijatović 2003]) to recognise any 3-manifold that satisfies the hypothesis of the theorem (just make all possible sequences of Pachner moves whose length is smaller than the bound!).

The unthinkable magnitude of this bound should, I suppose, be measured against the vastness of the class of 3-manifolds it covers. It for example gives a direct way of determining whether any 3-manifold is homeomorphic to a given nonfibred knot complement. In Section 3 we outline a simple procedure, based on Theorem 3.1 (which is a slight generalisation of Theorem 1.1), that can be used to decide if a knot, represented by a knot diagram, is the same as our given nonfibred knot.

The proof of Theorem 3.1 is carried out in several stages and uses a variety of techniques. The goal is to find an explicit bounded sequence of Pachner moves going from one triangulation to the other. We start by subdividing the original 
triangulation so that the characteristic submanifold is supported by a subcomplex of the subdivision. In other words, we start by constructing a triangulation of each piece of the JSJ-decomposition of our 3-manifold. The basic idea is to use the topological properties of the pieces to find the bounded sequence of Pachner moves in each of them.

The distinguished hierarchy techniques (see Section 4), together with Theorem 1.2 of [Mijatović 2003], are applied to the strongly simple pieces of the JSJdecomposition. This strategy amounts to building a two-dimensional polyhedron, called the distinguished hierarchy, with the property that its complement in our piece consists of embedded 3-balls. The additional hypothesis in Theorem 1.1 on the topology of the simple pieces of the JSJ-decomposition is crucial here, because without it we wouldn't be able to build our distinguished object. Once we have it, with the aid of Theorem 1.2 of [Mijatović 2003], it is possible to find a bounded sequence of Pachner moves connecting any two triangulations of the piece. The simplicial structure of the characteristic submanifold, which was obtained by subdividing the original triangulation of $M$, can be altered directly by applying the main theorem of [Mijatović 2004].

In Section 2 we give a brief exposition of JSJ-theory, generalise it to the setting of 3-manifolds with boundary pattern and prove a bound on the normal complexity of the canonical surfaces. Section 3 gives a precise definition of fibre-free 3-manifolds and states our main theorem. In Section 4 we define the distinguished hierarchy and prove that it has all the required properties. The last section puts everything together and proves Theorem 3.1.

\section{Canonical decompositions of 3-manifolds}

Any 3-manifold contains a (possibly empty) collection of canonical tori and annuli. Cutting along these surfaces we obtain a canonical decomposition of our space. These so-called JSJ-decompositions of 3-manifolds are due to Jaco and Shalen [1979] and Johannson [1979], with ideas from Waldhausen. When studying a triangulation of a Haken 3-manifold, it is profitable to make it interact well with the pieces of the JSJ-decomposition. In other words, the first step toward simplifying the triangulation of our 3-manifold will consist of subdividing the original triangulation so that the pieces of the JSJ-decomposition are triangulated by the subcomplexes of the subdivision.

In Section 2A we define canonical surfaces, JSJ-decompositions and characteristic submanifolds. The main reference for this is [Neumann and Swarup 1997]. Then, in Section 2B, we will study the parallel theory of canonical annuli in the 
presence of boundary patterns. This will be very useful when analysing the topology of hyperbolic pieces of our 3-manifold (see Section 4). In Section $2 \mathrm{C}$ we construct the canonical tori and annuli from fundamental surfaces.

2A. JSJ-decomposition and the characteristic submanifold. Consider an irreducible 3-manifold $M$ with (possibly empty) incompressible boundary. Let $F$ be a subsurface of $\partial M$. Recall that a properly embedded annulus or torus in the pair $(M, F)$ is called essential if it is incompressible and not parallel to an annulus or torus in $F$. The manifold $M$ is atoroidal if the pair $(M, \partial M)$ contains no essential tori, and an-annular if $(M, \partial M)$ contains no essential annuli. An essential annulus or torus $S$ is called canonical if any other essential annulus or torus in $(M, \partial M)$ can be isotoped to be disjoint from $S$.

Now we want to look at a disjoint maximal collection $\left\{S_{1}, \ldots, S_{k}\right\}$ of canonical surfaces in $M$ such that no two of the $S_{i}$ are parallel. Since we are assuming that $\partial M$ is incompressible in $M$, the essential annuli in $\left\{S_{1}, \ldots, S_{k}\right\}$ are also boundary incompressible. So a straightforward application of the Kneser-Haken finiteness theorem (see Theorem III.20 in [Jaco 1980]) guarantees the existence of such a maximal collection. The result of cutting $M$ along such a union of canonical surfaces is sometimes referred to as a Waldhausen decomposition of $M$. It is shown in [Neumann and Swarup 1997, Lemma 2.2] that a maximal system of disjoint canonical surfaces $\left\{S_{1}, \ldots, S_{k}\right\}$ is unique up to isotopy. In other words, any incompressible annulus or torus $S$ in $(M, \partial M)$ can be isotoped to be disjoint from the surface $S_{1} \cup \cdots \cup S_{k}$. Moreover, if $S$ is not parallel to any $S_{i}$, its final position in $M$-int $\mathcal{N}\left(S_{1} \cup \cdots \cup S_{k}\right)$ is determined up to isotopy, where $\mathcal{N}($ ) denotes a regular neighborhood.

Let's now look at a piece $M^{\prime}$ of the Waldhausen decomposition of $M$. Put differently, $M^{\prime}$ is simply a component of the cut-open manifold

$$
M-\operatorname{int} \mathcal{N}\left(S_{1} \cup \cdots \cup S_{k}\right) \text {. }
$$

Let $\partial_{1} M^{\prime}$ be the part of $\partial M^{\prime}$ coming from the surface $S_{1} \cup \cdots \cup S_{k}$ and let $\partial_{0} M^{\prime}$ equal $M^{\prime} \cap \partial M$. Clearly the union of $\partial_{0} M^{\prime}$ and $\partial_{1} M^{\prime}$ equals $\partial M^{\prime}$ and the components of $\partial_{1} M^{\prime}$ are annuli and tori. Also both surfaces $\partial_{0} M^{\prime}$ and $\partial_{1} M^{\prime}$ are incompressible, whereas it is possible for $\partial M^{\prime}$ to compress into $M^{\prime}$. We say that a piece $M^{\prime}$ is simple if any essential annulus or torus in $\left(M^{\prime}, \partial_{0} M^{\prime}\right)$ is parallel to a component of $\partial_{1} M^{\prime}$. If $M^{\prime}$ is a simple piece not admitting an incompressible annulus which is properly embedded in $\left(M^{\prime}, \partial_{1} M^{\prime}\right)$, then we call it strongly simple. It turns out that all the pieces that are simple but not strongly simple are either Seifert fibred or of the form (torus) $\times I$ with $\partial_{1} M^{\prime}=$ (torus) $\times \partial I$ (the manifold $M$ is in this case homeomorphic to a torus bundle over a circle with holonomy of trace different from \pm 2 ). The simple Seifert fibred pieces are of course very restricted as well; see [Neumann 
and Swarup 1997, Proposition 3.2 and Figure 1]. It is the topology of strongly simple pieces from our 3-manifold $M$ that we will be exploring in Section 4. The central result of the JSJ-theory [Neumann and Swarup 1997, Proposition 3.2] says that each complementary piece $M^{\prime}$ falls into one of the three categories:

(a) $\left(M^{\prime}, \partial_{0} M^{\prime}\right)$ is strongly simple.

(b) $\left(M^{\prime}, \partial_{0} M^{\prime}\right)$ is an $I$-bundle over a (possibly closed) surface.

(c) $\left(M^{\prime}, \partial_{0} M^{\prime}\right)$ is a Seifert fibred space with a possibly nonorientable base space.

These possibilities are almost mutually exclusive. Assuming that $\partial_{1} M^{\prime}$ is not empty, $M^{\prime}$ cannot be both strongly simple and Seifert fibred. The only $I$-bundles that are also strongly simple are the ones over the twice punctured disc and the once punctured Möbius band. The only $I$-bundle that is also Seifert fibred is the one over a Möbius band (see [Neumann and Swarup 1997, Proposition 3.3] for these nonuniqueness statements).

A canonical annulus in the 3-manifold $M$ separating two fibred pieces whose fibrations match along it, or separating a fibred piece from itself so that the fibrations on both sides of the annulus still match, is called a matching annulus. Notice that a piece $M^{\prime}$ of the Waldhausen decomposition containing a matching annulus has to be Seifert fibred, because an annulus separating two $I$-bundles is never canonical and hence cannot be a matching annulus. It also not hard to see that a canonical torus cannot separate two pieces with matching fibrations. Matching annuli do exist [Neumann and Swarup 1997, Lemma 3.4], but they carry no topological information. By deleting all matching annuli from our disjoint maximal collection of canonical surfaces $\left\{S_{1}, \ldots, S_{k}\right\}$ we obtain the JSJ-system of canonical surfaces for $M$. The decomposition of $M$ along this JSJ-system is called the JSJ-decomposition of $M$. Its pieces still fall into the three categories mentioned above.

Let's define a submanifold $\Sigma$ of $M$ in the following way. Let $\Sigma$ be a union of the $I$-bundle pieces and the Seifert fibred pieces of the JSJ-splitting of $M$. Let it also contain a regular neighbourhood of every canonical annulus or torus that separates two strongly simple pieces of the JSJ-decomposition, neither of which is an $I$-bundle. If two pieces of the JSJ-splitting of $M$ are already contained in $\Sigma$ and meet along a canonical torus or annulus, we remove the interiors of their regular neighbourhoods from $\Sigma$. The submanifold $\Sigma$ defined in this way is called the characteristic submanifold of $M$. Notice that the tori and annuli whose regular neighbourhoods we have removed from $\Sigma$ when defining it are precisely the ones along which two nonmatching fibred pieces meet (e.g., a Seifert fibred piece and an $I$-bundle). It also follows directly from the definition that every essential annulus or torus in $M$ can be isotoped into the characteristic submanifold $\Sigma$. 
2B. JSJ-theory with boundary pattern. Here and throughout this paper we will assume familiarity with the contents of [Mijatović 2004, Section 2]. This amounts to the basic definitions of incompressibility and $\partial$-incompressibility of embedded surfaces as well as some normal surface theory. We now define a key concept for the construction of the distinguished hierarchy.

A boundary pattern $P$ in a compact 3-manifold $M$ is a (possibly empty) collection of disjoint simple closed curves and trivalent graphs embedded in $\partial M$ such that the surface $\partial M-P$ is incompressible in $M$. Assume from now on that our 3-manifold $M$ is equipped with a boundary pattern $P$. If $M$ contains a properly embedded two-sided incompressible surface $S$ with $\partial S$ intersecting $P$ transversally (and missing the vertices of $P$ ), the cut-open manifold $M_{S}$ naturally inherits a boundary pattern as follows. If $S^{\prime}$ and $S^{\prime \prime}$ are the two copies of $S$ in $\partial M_{S}$, then the new boundary pattern, lying in $\partial M_{S}$, can be defined as $\left(P \cap \partial M_{S}\right) \cup \partial S^{\prime} \cup \partial S^{\prime \prime}$. Our definition of a boundary pattern implies that the manifold $M$ has incompressible boundary if and only if it admits an empty boundary pattern.

We shall use boundary patterns to keep track of the topological information as we move down the distinguished hierarchy (see Section 4). This is precisely the idea that Haken exploited to find an algorithm for classifying nonfibred 3-manifolds that contain an injective surface. At the heart of Haken's classification program lies the concept of a $P$-canonical annulus, which shall be described shortly.

Let $M$ be a 3-manifold with nonempty boundary that contains a boundary pattern $P$. Recall that a subset of $M$ is called pure if it has empty intersection with the pattern $P$. Most concepts from general 3-manifolds carry over to 3-manifolds with pattern in a very natural way. For example, a properly embedded surface $F$ in $M$ is $P$-boundary incompressible if for any pure disc $D$ in $M$ such that

$$
D \cap(\partial M \cup F)=\partial D
$$

and $D \cap F$ is a single arc in $F$, the arc $D \cap F$ cuts off a pure disc from $F$. Our definitions imply that a pure incompressible annulus in $M$ is $P$-boundary incompressible if and only if it is not parallel to a pure annulus in $\partial M$. A $P$-boundary incompressible pure annulus $A$ in $M$ will be called trivial if it is parallel (rel $\partial A$ ) to an annulus in the boundary of $M$. The interior of this annulus in $\partial M$ that $A$ is parallel to must have a nonempty intersection with the pattern $P$. Furthermore no spanning arc of this annulus in $\partial M$ can be pure. All this follows from the definition of the boundary pattern $P$ and the fact that $A$ is $P$-boundary incompressible. Also, an incompressible $P$-boundary incompressible pure annulus in $M$ is termed $P$ essential. So, according to our definitions, a $P$-essential annulus can be trivial. The next concept is of great importance in all that follows.

Definition. A properly embedded annulus $A$ in $M$ is a $P$-canonical annulus if it is nontrivial and $P$-essential in $M$ and has the following properties: 
- Any $P$-essential annulus in $M$ can be isotoped off of $A$ by an ambient isotopy that is invariant on the pattern $P$.

- Any incompressible torus not parallel to a (possibly nonpure) boundary component of $M$ can be isotoped off of $A$ by an isotopy that is fixed on the boundary of $M$.

The notion of a $P$-canonical annulus in 3-manifolds with pattern is a direct generalisation of the notion of a canonical annulus coming from JSJ-theory. It will be useful in Section 4 to observe that a solid torus with some pattern on its boundary can contain a $P$-essential annulus, but this annulus has to be trivial and therefore not $P$-canonical. Or more generally, a $P$-canonical annulus in $M$ cannot be parallel to an annulus in the boundary of $M$.

The next theorem will play a key role in the construction of the distinguished hierarchy (see Section 4). Its proof is a good example of how the ideas from JSJtheory generalise naturally to 3-manifolds with pattern.

Theorem 2.1. Let $M$ be an irreducible atoroidal 3-manifold with boundary pattern $P$. If $M$ contains a nontrivial $P$-essential annulus but no $P$-canonical one, then either of the two possibilities must occur:

(a) $M$ is homeomorphic to an I-bundle over a (possibly nonorientable) compact surface $F$, and its horizontal boundary ( $\partial$ I-bundle over $F$ ) is pure. The pattern $P$ is contained in the vertical boundary ( $I$-bundle over $\partial F$ ) and no fibre over any point in $\partial F$ can be pure.

(b) The manifold $M$ is an atoroidal Seifert fibred space. (For an explicit description see Figure 3 in [Mijatović 2004].)

Proof. We first take a maximal collection $\left\{A_{1}, \ldots, A_{k}\right\}$ of disjoint nonparallel $P$-essential annuli in $M$ (two pure annuli are parallel if they are parallel in the usual sense and the parallelism region between them is pure). Since we can assume that $M$ is triangulated and that the pattern $P$ is contained in the 1 -skeleton of that triangulation, any collection of disjoint $P$-essential annuli can be put into normal form. So our maximal collection of disjoint $P$-essential annuli exists by the Kneser-Haken finiteness theorem. Also, by assumption, we can make sure that at least one of the annuli $A_{i}$ is nontrivial. Let $N$ be a component of the cut-open manifold $M-\operatorname{int} \mathcal{N}\left(A_{1} \cup \cdots \cup A_{k}\right)$. We will show that $N$ fibres either as an $I$ bundle or as a Seifert fibred space. Moreover these fibrations will match up when we reglue the pieces to form the manifold $M$ we started with. The atoroidality of $M$ will impose severe restrictions on the Seifert fibred spaces that can arise. Most of the statements above will follow from the next claim.

Claim. The manifold $N$ is either homeomorphic to a pure I-bundle over an annulus, a punctured annulus, a Möbius band, or a punctured Möbius band or it is 
homeomorphic to a Seifert fibred space over a base surface from Figure 2. The pattern on the toral component of $N \cap \partial M$ in Figure $2 \gamma$ intersects every fibre of the Seifert fibration that is contained in that torus. All annular components of $N \cap \partial M$ in all the cases are pure except when the exceptional fibre in Figure $2 \beta$ is not singular. Then the annulus from $N \cap \partial M$ must contain components of pattern $P$ and no spanning arc of that annulus can be disjoint from the pattern.
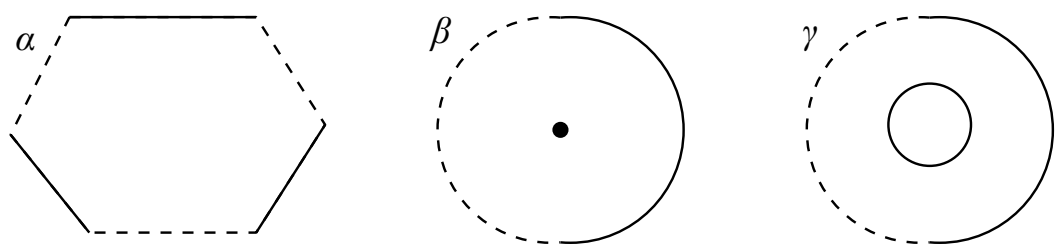

Figure 2. The Seifert fibred pieces that can arise as the manifold $N$. The solid and dashed parts of the boundary of the base surfaces correspond, respectively, to the part of the boundary of $N$ coming from $N \cap \partial M$ and $N \cap \partial \mathcal{N}\left(A_{1} \cup \cdots \cup A_{k}\right)$. The dot in case $\beta$ represents a fibre that may or may not be singular.

Before proving the claim, we note that if $N$ is a pure $I$-bundle over an annulus, $M$ has to be an $I$-bundle over a torus or a Klein bottle with empty pattern, and the theorem follows. This is because $M$ contains no $P$-canonical annuli and also no two annuli in $\left\{A_{1}, \ldots, A_{k}\right\}$ are parallel.

We are assuming that none of the $P$-essential annuli $A_{i}$ are $P$-canonical. So, an annulus $A_{i}$ is either trivial or there exists another $P$-essential annulus or an essential torus $A_{i}^{\prime}$ in $M$, such that the intersection $A_{i} \cap A_{i}^{\prime}$ is minimal and nonempty. The first possibility gives a solid torus as in Figure $2 \beta$ with the boundary as described in the claim. The dot in this case represents a nonsingular fibre.

We can assume now that $N$ is not a solid torus as in Figure $2 \beta$ with no exceptional fibres (such a piece must exist in $M$ because $M$ contains at least one nontrivial $P$-essential annulus). This means that it contains in its boundary a copy of an annulus $A_{i}$ which is not trivial. We shall now analyse the intersection $A_{i} \cap A_{i}^{\prime}$ for the annulus $A_{i}$ that is contained in $\partial N$. The 1-manifold $A_{i} \cap A_{i}^{\prime}$ contains no trivial simple closed curves and no separating properly embedded arcs in either of the two surfaces (among other things here, we are using the fact that the surface $\partial M-P$ is incompressible in $M$ ). The intersection can therefore consist either of nontrivial simple closed curves in $A_{i}$ and $A_{i}^{\prime}$, or of spanning arcs if both of our surfaces are annuli. The atoroidality of $M$ will impose limitations on the former possibility. 
Let's first look at the latter case. We are therefore assuming that the surface $A_{i}^{\prime}$ is an annulus. The intersection $N \cap A_{i}^{\prime}$ consists of a disjoint union of discs of length 4 which inherit a natural product structure from the annulus $A_{i}^{\prime}$. In other words, they are of the form $I \times I$. Let $D \subset N$ be one of those discs which intersects $A_{i}$, and let $s$ be a component of $D \cap A_{i}$. The product structure on $D$ naturally decomposes $\partial D$ into four arcs, $s$ being one of them. The two segments adjacent to $s$ are contained in $\partial M$ and are disjoint from the pattern $P$. Let $t$ be the fourth segment of $\partial D$, lying somewhere in the surface $A_{1} \cup \cdots \cup A_{k}$, say in the component $A_{j}$. We distinguish two subcases, depending on whether $A_{j}$ equals $A_{i}$.

For the proof of the fact that these two subcases yield the $I$-bundle possibilities in our claim, we refer the reader to the proof of Cases A1 and A2 of [Neumann and Swarup 1997, Proposition 3.2]. It is worth bearing in mind that if any of the annuli constructed by cut-and-paste in the cited proof are compressible, then they have to be isotopically trivial (that is, they bound a cylinder in $N$ ). This is because both discs obtained by compressing this cut-and-paste annulus chop off two 3-balls from $M$. Neither of these 3-balls can contain a properly embedded incompressible annulus. They can therefore not be nested and our cut-and-paste annulus must be isotopically trivial.

Now let's look at the case where $A_{i} \cap A_{i}^{\prime}$ consists of nontrivial simple closed curves. The surface $A_{i}^{\prime}$ can either be a $P$-essential annulus or an essential torus. This will make $N$ into a Seifert fibred space as described by the claim. The intersection $N \cap A_{i}^{\prime}$ consists of a disjoint union of annuli. Let $A$ be one of these annuli that intersects $A_{i}$, and let $s$ be a simple closed curve component of $A_{i} \cap A$. Let $t$ be the other boundary component of $A$. We have three possibilities, according to the position of the simple closed curve $t$.

If $t$ is contained in $N \cap \partial M$, then cutting $A_{i}$ along $s$ and pasting parallel copies of $A$ to the resulting annuli gives a pair of pure annuli that have to be $P$-essential. They inject into $M$ because $A_{i}$ injects. If either of them were $P$-boundary compressible, we could have reduced the number of components of the intersection $A_{i} \cap A_{i}^{\prime}$. They are therefore parallel to components of $A_{1} \cup \cdots \cup A_{k}$, which gives Figure $2 \alpha$.

If $t$ is contained in $A_{j}$ and $j$ is different from $i$, cutting and pasting as before gives two incompressible annuli that cannot both be $P$-boundary compressible (that would make $A_{i}$ and $A_{j}$ parallel). They cannot both be parallel into $\left\{A_{1}, \ldots, A_{k}\right\}$ (that is, parallel to elements of this collection), because that would contradict the maximality of the family $\left\{A_{1}, \ldots, A_{k}\right\}$. Thus one is parallel into $N \cap \partial M$ and the other to an annulus from $N \cap \mathcal{N}\left(A_{1} \cup \cdots \cup A_{k}\right)$. This gives Figure $2 \alpha$ again.

If $t$ lies in $A_{i}$, we have two further subcases, coming from $A$ meeting $A_{i}$ from the same side or from the opposite sides. The latter possibility gives the same as the previous case, except for one extra case when both annuli produced by cutand-paste are parallel into $N \cap \partial M$. This leads to $N$ being an $I$-bundle over an 
annulus. The manifold $M$ is then an $I$-bundle over a torus or over a Klein bottle with empty boundary pattern.

If $A$ meets $A_{i}$ from the same side and the orientations on $s$ and $t$ coincide, then cutting and pasting as before yields a torus and an annulus. The torus has to be either boundary parallel or compressible since $N$ is atoroidal. The annulus cannot be parallel into $\left\{A_{1}, \ldots, A_{k}\right\}$, because that would give rise to a new $P$-essential annulus in $N$, contradicting the maximality of $\left\{A_{1}, \ldots, A_{k}\right\}$. So the annulus has to be $P$-boundary compressible. This gives possibilities $\beta$ and $\gamma$ from Figure 2. In case $\beta$ the exceptional fibre has to be singular, because otherwise we could reduce the number of components of the intersection $A_{i} \cap A_{i}^{\prime}$. Also, the pattern on the toral boundary component in Figure $2 \gamma$ has to be such that no fibre of the Seifert fibration on that torus is pure.

The last case, when $A$ meets $A_{i}$ from the same side but the orientations $s$ and $t$ do not coincide, cannot occur. Cut-and-paste as before gives an annulus which is parallel into either $N \cap \partial M$ or $\left\{A_{1}, \ldots, A_{k}\right\}$. In both cases $N$ is an $S^{1}$-bundle over a Möbius band with a part of its boundary coming from a pure annulus in $N \cap \partial M$. We can easily construct a pure annulus in $N$, running once around the Möbius band, contradicting the maximality of $\left\{A_{1}, \ldots, A_{k}\right\}$. This proves the claim.

It is clear that the only manifold pieces from the claim for which the fibration is not unique up to isotopy on their boundaries are an $I$-bundle over a Möbius band and an $I$-bundle over an annulus. The first one also fibres as a Seifert fibred space from Figure $2 \beta$ with a singular fibre of index $\frac{1}{2}$. The second we do not need to consider, because we already know that the theorem is true if such a piece appears. Since the nonunique piece $N$ has a single annulus coming from the collection $\left\{A_{1}, \ldots, A_{k}\right\}$, we can always extend uniquely the fibration on the boundary. Since we can do that for all other pieces of the complement $M-\operatorname{int} \mathcal{N}\left(A_{1} \cup \cdots \cup A_{k}\right)$ as well, it follows that $M$ is either an $I$-bundle with the described boundary pattern or a Seifert fibred space. But since the Seifert fibred space is also atoroidal, the theorem follows.

2C. Canonical surfaces in normal form. We now explain how to construct canonical tori and annuli in a triangulated 3-manifold using normal surface theory. Our proofs rely on the key notion of a trivial patch and are based on the following lemma and theorem (see Section 2 in [Mijatović 2004]):

Lemma 2.2. Let $M$ be an irreducible 3-manifold with a (possibly empty) boundary pattern $P$. Let $F$ be a incompressible P-boundary incompressible normal surface of minimal weight. If the sum $F=F_{1}+F_{2}$ is in reduced form then each patch is both incompressible and P-boundary incompressible and no patch is trivial. Furthermore, if $F$ is injective, each patch has to be injective. 
Theorem 2.3. Let $M$ be an irreducible 3-manifold with a possibly empty boundary pattern $P$. Let $F$ be a normal surface of least weight properly embedded in $M$. Assume also that $F$ is two-sided incompressible $P$-boundary incompressible and $F=F_{1}+F_{2}$. Then $F_{1}$ and $F_{2}$ are incompressible and P-boundary incompressible.

The next two propositions are going to be crucial when we subdivide the original triangulation of $M$. In particular we will find Proposition 2.5 (page 153) indispensable while deciphering the topology of the strongly simple pieces in the JSJdecomposition of $M$.

Proposition 2.4. Let $M$ be an irreducible 3-manifold with (possibly empty) incompressible boundary. Let $t$ be the number of tetrahedra in the triangulation $T$ of $M$. Then every canonical torus in $M$ can be isotoped into normal form so that it contains not more than $2^{80 t^{2}}$ normal discs. The same remains true if a single canonical torus in $M$ is replaced by a disjoint maximal collection of nonparallel canonical tori in $M$.

Proof. We start by taking our maximal collection $C$ of disjoint canonical tori in $M$ and putting it into normal form, so that it has the smallest weight in its isotopy class. The normal surface $C$ can then be expressed as a sum of fundamental surfaces $C=k_{1} F_{1}+\cdots+k_{n} F_{n}$, where $k_{1}, \ldots, k_{n}$ are positive integers.

Claim 1. Each surface $F_{i}$ is either an incompressible torus or an incompressible Klein bottle.

Proof. We get the incompressibility of $F_{i}$ by applying Theorem 2.3. All we need to do is fix a copy of the surface $F_{i}$ and make all regular alterations along curves of intersection (in the sum $k_{1} F_{1}+\cdots+k_{n} F_{n}$ ) that do not lie in our copy of $F_{i}$. This yields a sum $C=F_{i}+F^{\prime}$, where $F^{\prime}$ is some normal surface in $M$. Since both $C$ and $M$ are orientable, the surface $C$ is two-sided. Since the normal representative for $C$ has minimal weight, we can apply Theorem 2.3 to conclude that $F_{i}$ must be incompressible as well.

If we isotope the sum $C=F_{i}+F^{\prime}$ into reduced form, we can apply Lemma 2.2 to conclude that no patch can be trivial. This means that the surface $F_{i}$ cannot be a 2-sphere. We are assuming that $M$ contains at least one canonical torus and is therefore different from $\mathbb{R} P^{3}$. Since it is also irreducible, $F_{i}$ cannot be a projective plane either. Now all fundamental surfaces in our sum are closed and connected. So the claim follows by the additivity of the Euler characteristic.

Note that the proof of the claim uses only the incompressibility of $C$, not the fact that it is canonical. Also the same method of proof shows that any connected normal surface that appears as a summand of $C$ has to be an incompressible torus or an incompressible Klein bottle. 


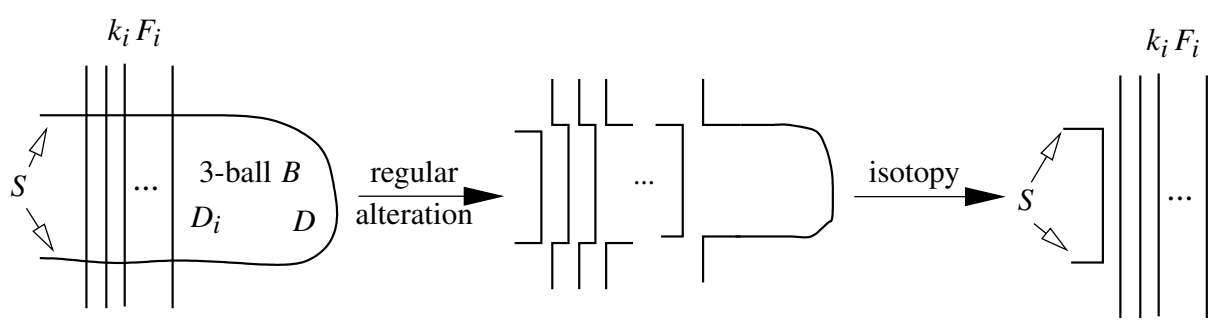

Figure 3. Regular alteration along the curve $\partial D_{i}$ and the isotopy across the 3-ball $B$.

We now want to bound the coefficients $k_{i}$ in the sum $C=k_{1} F_{1}+\cdots+k_{n} F_{n}$. After making all regular alterations in the sum above, except the ones on the surface $k_{i} F_{i}$, our expression can be rewritten as $C=k_{i} F_{i}+S$. The surface $S$ is a (possibly disconnected) closed incompressible normal surface with zero Euler characteristic.

Let's now investigate the patches of the polyhedron $K=F_{i} \cup S$, i.e., the surface components of the complement of the singular locus of $K$. It is clear that our surface $C$ lies in a regular neighbourhood of $K$. The plan is to alter $K$ in such a way that it contains no disc patches but is still a union of normal surfaces whose integral linear combination represents $C$. We want to do this in such a way that the weight of the new normal surfaces is not greater than that of $F_{i}$ and $S$.

If there exists a trivial patch $D_{i}$ in $F_{i}$ bounded by a simple closed curve from $F_{i} \cap S$, then this curve must bound a disc $D$ in $S$ distinct from $D_{i}$. We now look at each patch $D_{i}$ of $K$ that is contained in $F_{i}$, such that a copy of the disc $D$ is also a patch in $K$. In other words, the singular locus of $K$ intersects $D$ in its boundary only, making the disc $D$ into a patch in the surface $C$.

After making all regular alterations in the sum $k_{i} F_{i}+S$, one of the parallel copies of the disc $D_{i}$ is adjacent to the disc $D$ in the surface $C$ (see Figure 3). Since the manifold $M$ is irreducible, the union of discs $D \cup D_{i}$ bounds a 3-ball $B$ as in Figure 3. If the weights of the discs were not equal, we could use this 3-ball $B$ to isotope $C$ so that its weight is reduced. Thus we must have $w\left(D_{i}\right)=w(D)$.

Now we modify the surface $C$ by pushing the disc $D$ across the ball $B$. Notice that this operation did not change the weight of $C$. Also the surface we end up with is again in normal form. A similar kind of modification can be done to the surface $S$. In this case we push the disc $D$ so that it misses all parallel copies of $D_{i}$ but it is still normally parallel to the disc $D_{i}$. This produces a new normal surface $S^{\prime}$ which has the same weight as $S$. Also the normal equation $C=k_{i} F_{i}+S^{\prime}$ holds for the isotoped surface $C$.

We keep applying this same procedure to all patches of $K$ which are discs in $F_{i}$ and have adjacent trivial patches from $S$ next to them. In the end we obtain a polyhedron $K^{\prime}=F_{i} \cup S^{\prime}$ where $S^{\prime}$ is a normal surface with the property $w\left(S^{\prime}\right)=$ $w(S)$ and $K^{\prime}$ contains no pairs of adjacent disc patches. 
Claim 2. The polyhedron $K^{\prime}$ contains no patches which are discs.

Proof. Clearly it is enough to show that the sum $C=k_{i} F_{i}+S^{\prime}$ contains no trivial patches. Let's assume that there exists a trivial patch $R$ somewhere in $C$ and that it has the smallest weight among all trivial patches. Since $\chi\left(F_{i}\right)=\chi(S)=0$, the boundary of $R$ has to be two-sided in both summands. So there exists a unique disc $R^{\prime}$ in $C$ whose boundary is adjacent to that of $R$. Disc $R^{\prime}$ is different from the trivial patch $R$, but it might contain it. Also $R^{\prime}$ is not itself a patch, since $K^{\prime}$ contains no pairs of adjacent disc patches. We must have $w\left(R^{\prime}\right)=w(R)$; otherwise we could reduce the weight of $C$. Therefore $R^{\prime}$ contains precisely one trivial patch $Q$ with $w(Q)=w(R)$ and also other annular patches of zero weight. Now we take the patch $Q$ and repeat the procedure. We can use the same argument as in the proof of Lemma 3.6 in [Bart and Scharlemann 1996] to express the surface $C$ as a sum $C=C^{\prime}+B$, where $B$ is a normal surface with zero weight (compare the proof of Lemma 2.2 in [Mijatović 2004]). This contradiction proves the claim.

Claim 2 implies that all patches of $K^{\prime}$ are incompressible subsurfaces of $C$ with nonpositive Euler characteristic. Therefore they have to be incompressible annuli. So the regular neighbourhood $\mathcal{N}\left(K^{\prime}\right)$ supports a structure of a Seifert fibred space with no singular fibres. The surface $C$ is naturally contained in $\mathcal{N}\left(K^{\prime}\right)$ and can be viewed as a circle bundle over a disjoint union of simple closed curves in the base surface of our Seifert fibration.

The boundary $\partial \mathcal{N}\left(K^{\prime}\right)$ consists of tori. If a toral component of $\partial \mathcal{N}\left(K^{\prime}\right)$ is compressible in $M$, it either bounds a solid torus in $M$ (which is disjoint from $\mathcal{N}\left(K^{\prime}\right)$, because a solid torus cannot contain an incompressible torus) or it is contained in a 3-ball. The latter possibility cannot occur, because then some fibre from $\mathcal{N}\left(K^{\prime}\right)$ would be homotopically trivial, contradicting the incompressibility of patches of $K^{\prime}$. In the former case we can extend the Seifert fibration over the solid torus. We can always do that because the fibres in the boundary are homotopically nontrivial in $M$. Doing that for all compressible components of $\partial \mathcal{N}\left(K^{\prime}\right)$, we obtain a new Seifert fibred space $X$ which is embedded in $M$, contains $\mathcal{N}\left(K^{\prime}\right)$, and must have a nonempty incompressible boundary in $M$. If the 3-manifold $X$ were closed, it would have to be equal to the whole of $M$, making it into a closed Seifert fibred space containing vertical canonical tori. This is clearly a contradiction.

We should note at this point that the construction up to now was based on the fact that the surface $C$ is incompressible. The hypothesis that $C$ is in fact canonical will now come into play.

Assume that some simple closed curve in the base surface of $X$ is not boundary parallel in the base space, and that a toral component $C_{0}$ of $C$ fibres over this curve. Then we can find in the base surface another simple closed curve such that $C_{0}$ cannot be isotoped off the vertical torus above this curve by an isotopy supported 
in $X$. Because $\partial X$ is incompressible in $M$, so is the vertical torus. Hence, since $C_{0}$ is canonical, there exists a homotopy $h: C_{0} \times I \rightarrow M$ that will move $C_{0}$ so that it is disjoint from the vertical torus and from the boundary $\partial X$. The preimage of $\partial X$ under $h$ is a closed surface in the product $C_{0} \times I$. Using Dehn's lemma and the loop theorem we can modify the homotopy so that $h^{-1}(\partial X)$ becomes a disjoint union of copies of $C_{0}$ in the interior of the product $C_{0} \times I$. The fact that two homotopic embeddings of an incompressible surface are isotopic (see Corollary 5.5 in [Waldhausen 1968]) implies that we were isotoping $C_{0}$ over a boundary component of $X$ and that $C_{0}$ is parallel to it. But this contradicts our initial assumption. So the collection of simple closed curves in the base surface of $X$ that $C$ fibres over must be boundary parallel.

The classification of surfaces implies that there exists, in the base surface of the Seifert fibred space $X$, a family of disjoint annuli such that one boundary component of each annulus is a boundary curve of the base surface. The union of the other boundary components of our annuli is precisely the simple closed curves in the base surface of $X$ that the surface $C$ fibres over.

Even though the manifold $X$ can contain singular fibres, the family of annuli just described contains no singular points. This again follows from the fact that $C$ is canonical. If one of the annuli contained a singular point, we could easily construct an incompressible torus in $X$ that could not be isotoped off $C$.

\section{Claim 3. Each coefficient $k_{i}$ can either be 1 or 2.}

Proof. Let's assume that $k_{i}$ is larger than 2 and let's look at some annular patch of $F_{i}$ with nonzero weight. Since there are at least 3 copies of this patch contained in $C$, after using the projection of $X$ onto its base surface, we can conclude that the images of two adjacent copies of our patch belong to the same boundary component of one of the annuli constructed above. Furthermore there exists an arc $\alpha$, properly embedded in this annulus, running between the two adjacent copies of the projections of our patch. The arc $\alpha$ chops off a disc in the annulus, bounded by $\alpha$ on one side and an arc $\beta$ which is contained in the image of the projection of $C$. Now we can use this disc to isotope $\beta$ onto $\alpha$. Since our annulus contains no singular points, we can extend this isotopy, thus obtaining an isotopy of $C$, which pushes the annulus over $\beta$ onto the annulus over $\alpha$. By choosing the $\operatorname{arc} \alpha$ judiciously, we can make sure that this isotopy reduces the weight of $C$ since the patch of $F_{i}$ we started with had positive weight. But this is a contradiction because $C$ had minimal weight. So the claim follows.

The formula in Lemma 6.2 of [Hass et al. 1999] giving a bound on the number of all fundamental surfaces is bounded above by $2^{70 t^{2}}$. Using a well known bound on the normal complexity of fundamental surfaces (see [Hass and Lagarias 2001], 
for example) and the estimate $2 \cdot 20 t^{2} 2^{7 t} \leq 2^{10 t^{2}}$ for $t \geq 2$, we get the desired bound, proving Proposition 2.4.

Note that the proof only required the property that surfaces in $C$ are toruscanonical, i.e., that each torus in $C$ can be isotoped off any incompressible torus in $M$. This notion is very close to but not identical with the notion of a canonical surface. In fact an essential torus is torus-canonical if and only if it is either canonical or it is parallel to a torus formed from a canonical annulus and an annulus in $\partial M$ (see Proposition 4.2 in [Neumann and Swarup 1997]). This means that even if $M$ has nonempty pattern on its boundary, Proposition 2.4 still bounds the normal complexity of $P$-canonical tori in it.

Now we describe how to construct canonical annuli in triangulated 3-manifolds.

Proposition 2.5. Let $M$ be an irreducible 3-manifold with nonempty boundary. Assume that $M$ admits a possibly empty boundary pattern $P$ contained in the 1skeleton of the triangulation $T$ of $M$. Let $t$ be the number of tetrahedra in $T$. Then every $P$-canonical annulus in $M$ can be isotoped into normal form so that it consists of not more than $2^{80 t^{2}}$ normal discs. The same bound is valid if we replace a single P-canonical annulus with a maximal collection of disjoint topologically nonparallel $P$-canonical annuli.

Proof. Let $A$ be a maximal collection of disjoint topologically nonparallel $P$ canonical annuli in $M$. Without loss of generality we can assume that the surface $A$ is in normal form and that its weight is minimal. We can therefore express it as a sum of fundamental surfaces $A=k_{1} F_{1}+\cdots+k_{n} F_{n}$, where the coefficients $k_{i}$ are positive integers. Just as in the proof of Proposition 2.4, we can show that the surfaces $F_{i}$ are incompressible $P$-boundary incompressible and pure. By applying Lemma 2.2 in the same way as before, we can conclude that $\chi\left(F_{i}\right)$ is zero.

We now want to bound each integer $k_{i}$. As before we can express $A$ as a sum $A=k_{i} F_{i}+S$, where $S$ is a pure normal incompressible $P$-boundary incompressible surface in $M$ with zero Euler characteristic. Let's look at the polyhedron $K=F_{i} \cup S$. By using the procedure described in Figure 3 we can make sure that $K$ contains no adjacent trivial patches that are bounded by a simple closed curve from the intersection $F_{i} \cap S$. The same argument that justified Claim 2 from the previous proof tells us that after this modification, the polyhedron $K$ contains no trivial patches disjoint from $\partial M$. It might however contain trivial patches that do meet the boundary of $M$.

We start by looking at adjacent trivial patches in $K$, i.e., the ones that meet along an arc of intersection $F_{i} \cap S$ (any such pair of trivial patches cannot be contained in one of the surfaces $F_{i}$ or $S$, because both $F_{i}$ and $S$ are not a disc). By doing regular alterations in the sum $A=k_{i} F_{i}+S$ along each such arc from $F_{i} \cap S$ and then performing an isotopy that is invariant on the pattern $P$ we get a new normal 
surface $S^{\prime}$ such that $w(S)=w\left(S^{\prime}\right)$ (adjacent trivial patches must have the same weight because $A$ has minimal weight). Furthermore the equality $A=k_{i} F_{i}+S^{\prime}$ holds for the isotoped surface $A$ and the new polyhedron $K^{\prime}=F_{i} \cup S^{\prime}$ contains no adjacent trivial patches. It follows directly that the surface $A$ now contains no adjacent trivial patches either. In fact it contains no trivial patches at all. If it contained one, we could do the same construction as in the second part of the proof of [Mijatović 2004, Lemma 2.2] to obtain a normal sum $A=A^{\prime}+B$, where the normal surface $B$ misses the 1-skeleton. This contradiction, together with the fact that $F_{i}$ is connected, implies that all patches of $A$ are either annuli or discs of length four. The latter case means that the patches are discs bounded by four arcs, two of them in $\left(k_{i} F_{i}\right) \cap S^{\prime}$ and the other two in $\partial M$.

The former case, when the patches are annuli, is almost identical to the situation from the previous proposition. Again the regular neighbourhood $\mathcal{N}\left(K^{\prime}\right)$ supports a structure of a Seifert fibred space. The surface $A$ is vertical in this Seifert fibration and is therefore determined by a collection of properly embedded disjoint arcs in the base surface of the fibration. All compressible toral components of $\partial \mathcal{N}\left(K^{\prime}\right)$ that are disjoint from $\partial M$ have to bound solid tori in $M$ disjoint from $\mathcal{N}\left(K^{\prime}\right)$. This follows by the same argument as in the proof of Proposition 2.4. We can extend the fibration over all such solid tori. The annuli from $\partial \mathcal{N}\left(K^{\prime}\right)-\partial M$ are incompressible simply because their core curves are generators of the fundamental group of $A$. If such an annulus is $P$-boundary compressible, then it is parallel to a pure annulus in $\partial M$. This parallelism region is topologically a solid torus. We can extend the existing fibration of our manifold over it. After we have adjoined all such parallelism regions to our submanifold, we obtain a Seifert fibred space $X$ that contains $A$ as a vertical surface.

As in the proof of Proposition 2.4, we can show that any vertical torus in $X$ that is not parallel to a component of $\partial M$ can be isotoped off $A$ by an isotopy that is supported in $X$. Since the tori from $\partial X$ are incompressible in $M$, we have two possibilities. Either we are able to isotope $A$ off all of the essential vertical tori in $X$ or we are not. In the former case the annuli of $A$ are boundary parallel in $X$. In other words, their projection onto the base surface of $X$ chops off from the base surface discs that contain no singular points. We can therefore use the same technique that proved Claim 3 from the proof of Proposition 2.4 to bound each coefficient $k_{i}$. In the latter case there exists at least one annulus $A_{0}$ in $A$ that lies in a component $X_{0}$ of $X$ which is homeomorphic to one of the atoroidal Seifert fibred spaces (see Figure 3 in [Mijatović 2004]). This is because $X_{0}$ cannot have any essential vertical tori. Also, since the surface $\partial X$ is incompressible in $M$, the boundary components of $X_{0}$ that contain $\partial A_{0}$ must be parallel to components of $\partial M$. The components of $\partial X_{0}$ that are disjoint from $A_{0}$ are not necessarily parallel into $\partial M$ and can be canonical tori in $M$. In this topological setting it is not hard 
to prove, using our trivial patch reduction techniques and Theorem 2.3, that $A_{0}$ is actually fundamental in $M$ and that it therefore satisfies Proposition 2.5.

We can now assume that all patches of $A$ are discs of length four. This implies that the regular neighbourhood $\mathcal{N}\left(K^{\prime}\right)$ supports an $I$-bundle structure. The boundary of the manifold $\mathcal{N}\left(K^{\prime}\right)$ is naturally divided into two bits. The horizontal part, which is contained in $\partial M$, is just a $\partial I$-bundle. The vertical part is a complement (in $\partial \mathcal{N}\left(K^{\prime}\right)$ ) of the horizontal boundary and it consists of properly embedded pure annuli in $M$.

Claim. If an annulus $V$ from the vertical boundary of $\mathcal{N}\left(K^{\prime}\right)$ is compressible, it bounds a pure submanifold of the form $D^{2} \times I$ in $M$, such that $\left(D^{2} \times I\right) \cap \partial M=$ $D^{2} \times \partial I$ and $\left(D^{2} \times I\right) \cap V=\partial D^{2} \times I$. This submanifold is disjoint from $A$.

Proof. By compressing the annulus $V$ we obtain two pure properly embedded discs $D_{1}^{\prime}$ and $D_{2}^{\prime}$ in $M$. From the the definition of the pattern $P$ and the fact that $M$ is irreducible it follows that $D_{1}^{\prime}$ and $D_{2}^{\prime}$ are parallel to discs $D_{1}$ and $D_{2}$ in $\partial M$. The parallelism region between the discs $D_{i}$ and $D_{i}^{\prime}$ is a pure 3-ball $B_{i}$ for $i=1,2$. Then there are two possibilities.

If $B_{1}$ and $B_{2}$ are disjoint, the discs $D_{1}$ and $D_{2}$ are also disjoint and the 2-sphere $D_{1} \cup D_{2} \cup V$ bounds a 3-ball with the required properties. Also $A$ has to be disjoint from this 3-ball because it consists of incompressible annuli. So the claim follows in this case.

If $B_{1}$ and $B_{2}$ are nested, we can assume that $B_{1}$ is contained in $B_{2}$, so $D_{1}$ lies in int $D_{2}$. The surface $A$ is disjoint from $V$ and hence does not meet the boundary circles $\partial V=\partial D_{1} \cup \partial D_{2}$. Since $A$ consists of incompressible annuli, both $A \cap D_{1}$ and $A \cap D_{2}$ must be empty. But it follows from the definition of $V$ that there exists an embedded arc in $\partial M$, running from $\partial D_{1}$ to $\partial A$, which is disjoint from $\partial D_{2}$. In other words, $A \cap$ int $D_{2}$ cannot be empty. This contradiction proves the claim.

We can now extend the $I$-bundle structure of $\mathcal{N}\left(K^{\prime}\right)$ over every compressible vertical annulus in the boundary of $\mathcal{N}\left(K^{\prime}\right)$. If an incompressible vertical annulus is $P$-boundary compressible, then it has to be parallel to a pure annulus in $\partial M$. The parallelism region, which is a solid torus, can be used to construct a pure $\partial$ compression disc for some annulus in $A$. So this cannot arise. After adjoining all the solid cylinders to $\mathcal{N}\left(K^{\prime}\right)$, we obtain a pure $I$-bundle $X$ in $M$ whose vertical boundary consists of incompressible $P$-boundary incompressible annuli. The horizontal boundary of $X$ is a pure subsurface of $\partial M$. The collection of vertical annuli in $\partial X$ is not empty, since otherwise $M$ would have to be homeomorphic to an $I$-bundle over a closed (possibly nonorientable) surface with an empty boundary pattern. But such manifolds contain no canonical annuli.

We now apply the fact that the annuli in $A$ are $P$-canonical. It follows, in the same way as before, that $A$ can be isotoped off any annulus in $X$ that fibres over a 
simple closed curve in the base surface of $X$, by an isotopy that is supported in $X$. This implies that the components of $A$ have to be parallel to the vertical annuli from $\partial X$. So we can use the same strategy as in Claim 3 from the proof of Proposition 2.4 to show that the coefficient $k_{i}$ cannot exceed 2 . The only difference is that the weight-reducing isotopy is supported in a pure solid cylinder rather than in a solid torus. Also the same bounds on the number of normal discs, as in the proof of Proposition 2.4, are valid here. This concludes the proof of Proposition 2.5.

It is probably true that the bound on normal complexity of canonical surfaces given by Propositions 2.4 and 2.5 is not the best possible. On the other hand it is not hard to construct simple closed curves in a bounded surface, none of which is boundary parallel, but whose "normal" sum is boundary parallel. The $S^{1}$-bundle over such a surface can be a component of the characteristic submanifold in some ambient 3-manifold. The boundary torus of the $S^{1}$-bundle is certainly not going to be fundamental, but in this setting it is clearly canonical.

It is worth pointing out that Propositions 2.4 and 2.5 together imply the same bound on the normal complexity of the whole JSJ-system of surfaces in $M$.

The proof of Proposition 2.5 clearly bounds the normal complexity of every trivial $P$-essential annulus in $M$. This is because such an annulus can also be isotoped off of any other $P$-essential annulus in $M$. But it is not hard to show that, in an atoroidal 3-manifold where all $P$-essential annuli are trivial and which is not a solid torus, every trivial $P$-essential annulus is isotopic to a fundamental annulus. First we isotope it so that it has minimal weight. If it is a sum of two surfaces, we can assume that they are connected. By applying Lemma 2.2 we can conclude that both of them have zero Euler characteristic, since they are both pure. By Theorem 2.3 they are both incompressible and $P$-boundary incompressible. At least one of the summands is bounded. It cannot be a Möbius band because our manifold is not a solid torus. So it is a trivial $P$-essential annulus which can be isotoped off of our original annulus by an isotopy that is invariant on the pattern. If the other summand is closed, then these two annuli have to be parallel. This contradicts our minimal-weight assumption on the original trivial $P$-essential annulus. If the other summand is also a trivial $P$-essential annulus then, after removing all trivial patches in the normal sum, we can conclude that both of the summands have to be parallel to our original annulus. This contradiction proves our claim.

\section{Statement of the Main Theorem}

In order to state Theorem 3.1 we need to clarify what we mean by a fibre-free 3manifold. We start by recalling some standard terminology. A surface bundle with an orientable fibre $S$ is just a mapping torus, i.e., a quotient $S \times I /(x, 0) \sim(\varphi(x), 1)$, for some orientation-preserving surface automorphism $\varphi: S \rightarrow S$. Since $S$ is 
orientable this construction gives an orientable 3-manifold. But for a nonorientable surface $R$ with a nontrivial two-sheeted covering $S \rightarrow R$, the mapping cylinder of the covering projection is an orientable twisted $I$-bundle over $R$. Gluing two such $I$-bundles together along their horizontal boundaries by an automorphism of $S$ gives a 3-manifold $N$ foliated by parallel copies of $S$ and the two copies of $R$. The leaves of this foliation are the "fibres" of a natural projection map $N \rightarrow I$, where the two copies of $R$ are the preimages of the endpoints of the interval $I$. Such a 3-manifold $N$ will be called a semibundle (with fibre $S$ ) over an interval $I$. The surfaces $S$ and $R$ can be either closed or bounded.

Manifolds which are semibundles do sometimes arise in nature. For example, a Klein bottle is a semibundle with fibre $S^{1}$, since it splits as a union of two Möbius bands. The simplest example in dimension 3 is a connected sum of two projective spaces $\mathbb{R} P^{3} \# \mathbb{R} P^{3}$, where the fibre is a 2 -sphere. On the other hand, a semibundle structure can never arise in a knot complement. This is because the boundary circles of the two nonorientable leaves would be disjoint curves in the boundary torus and could therefore be capped off by the annuli they bound. This would then give a closed nonorientable surface in $S^{3}$.

Any semibundle over an interval can be viewed as a quotient of the product $S \times I$ with identifications $(x, 0) \sim(\alpha(x), 0)$ and $(x, 1) \sim(\beta(x), 1)$. The homeomorphisms $\alpha$ and $\beta$ are orientation-reversing fixed-point-free involutions of the fibre $S$. Using this representation it is easy to see that every semibundle admits a two-sheeted covering space that is a surface bundle. The holonomy of the surface bundle is the composition of $\alpha$ and $\beta$. So for example every semibundle, except the one mentioned above, is irreducible, because the surface bundle covering it is covered by $\mathbb{R}^{3}$, which is irreducible.

Recall that a Haken 3-manifold is an irreducible 3-manifold with possibly empty incompressible boundary and containing an injective surface different from a disc or a 2-sphere. We now define the class of 3-manifolds considered in Theorem 3.1.

Definition. A 3-manifold $M$ with a nontrivial JSJ-decomposition or with nonempty boundary is fibre-free if none of the strongly simple pieces in its JSJ-decomposition are homeomorphic to a surface bundle or semibundle containing no closed injective surfaces. A closed atoroidal Haken 3-manifold is fibre-free if it is not homeomorphic to a closed surface bundle over $S^{1}$ or to a closed surface semibundle over $I$.

Thus a fibre-free 3-manifold $M$ can contain a bounded surface bundle or semibundle if it is a part of the characteristic submanifold $\Sigma$ or if it contains a closed injective surface. In other words, if, say, a surface-bundle in a JSJ-decomposition of $M$ supports a Seifert fibration, then the manifold $M$ is still fibre-free. It is known that, if the surface-fibre has negative Euler characteristic, this happens if and only if 
the holonomy is a periodic element of the mapping-class group of the surface-fibre; see Lemma VI.31 in [Jaco 1980].

Theorem 3.1. Let $M$ be a fibre-free Haken 3-manifold. Let $P$ and $Q$ be two triangulations of $M$ that contain $p$ and $q$ tetrahedra respectively. Then there exists a sequence of Pachner moves of length at most $E^{2^{a p}}(p)+E^{2^{a q}}(q)$ (see Convention on page 140) that transforms $P$ into a triangulation isomorphic to $Q$. The constant $a$ is bounded above by 200. The homeomorphism of $M$, that realizes this simplicial isomorphism, is supported in the characteristic submanifold $\Sigma$ of $M$ and it does not permute the components of $\partial M$.

This theorem gives a conceptually trivial algorithm for determining whether any 3-manifold is homeomorphic to a complement of a given nonfibred knot in the 3sphere. Say we also wanted a simple procedure enabling us to determine whether any knot is the same as our given nonfibred knot. It is enough to establish whether their respective complements are homeomorphic and, if they are, to determine if the homeomorphism maps the meridian of one onto the meridian of the other (the last requirement is a direct consequence of the famous result of Gordon and Luecke [1989], but we would like to stress here that our algorithm works without this complicated and deep result). If the boundary torus of this knot complement is not contained in the characteristic submanifold, then the homeomorphism from Theorem 3.1 equals the identity on the boundary. If on the other hand the bounding torus is contained in $\Sigma$, we first make sure that the simplicial structures on the boundary of both knot complements coincide. It will be clear from the proof of Theorem 3.1 that this makes the homeomorphism equal to the identity on the boundary torus. So in this way, using Theorem 3.1, we can solve the recognition problem for any nonfibred knot.

The proof of Theorem 3.1 starts by subdividing the original triangulation of $M$ so that the characteristic submanifold $\Sigma$ is triangulated by a subcomplex of the subdivision. We then look at the strongly simple pieces of the JSJ-decomposition. We will simplify their triangulations using the distinguished hierarchy which is described in Section 4. The distinguished triangulation is obtained from the distinguished hierarchy by applying Theorem 1.2 from [Mijatović 2003]. So a triangulation of every strongly simple piece will impose a simplicial structure in the boundary of both Seifert fibred and $I$-bundle components of $\Sigma$. The former can then be simplified by Theorem 3.1 of [Mijatović 2004], and the latter can be dealt with using techniques from [Mijatović 2004, Section 6.2] and from Section 4B.

\section{The distinguished hierarchy}

Let $M$ be a 3-manifold satisfying the hypothesis of Theorem 3.1. In this section we describe the distinguished two-dimensional object lying inside $M$ that will give 
rise to an intermediate triangulation, later to be used to bridge the gap between any two triangulations of $M$. Let's start by introducing some standard terminology. Let $S$ be an incompressible surface contained in the 3-manifold $M$. The operation of cutting $M$ along $S$ results in a 3-manifold $M_{S}$ that is just a complement (in $M$ ) of the interior of the regular neighbourhood $\mathcal{N}(S)$.

Definition. A partial hierarchy for a Haken 3-manifold $M$ is a sequence of 3manifolds $M_{1}, \ldots, M_{n}$, where $M_{1}$ equals $M$ and $M_{i+1}$ is obtained from $M_{i}$ by a cutting along an orientable, incompressible, properly embedded surface in $M_{i}$, no component of which is a 2-sphere. A hierarchy is a partial hierarchy with the property that $M_{n}$ is a collection of 3-balls. We shall denote (partial) hierarchies by, for example,

$$
M_{1} \stackrel{S_{1}}{\longrightarrow} M_{2} \stackrel{S_{2}}{\longrightarrow} \cdots \stackrel{S_{n-2}}{\longrightarrow} M_{n-1} \stackrel{S_{n-1}}{\longrightarrow} M_{n},
$$

where $S_{i}$ is the surface in $M_{i}$ that we cut along.

It is well known that every Haken 3-manifold possesses a hierarchy. Since we are going to construct a hierarchy with some additional properties we will be, among other things, reproving this result. Another classical fact about hierarchies is contained in Lemma 4.1. Since it represents a key step in the construction of the distinguished hierarchy, we will include its proof.

Lemma 4.1. Let $M$ be a compact orientable irreducible 3-manifold with (possibly empty) boundary. Let

$$
M_{1} \stackrel{S_{1}}{\longrightarrow} M_{2} \stackrel{S_{2}}{\longrightarrow} \cdots \stackrel{S_{k-2}}{\longrightarrow} M_{k-1} \stackrel{S_{k-1}}{\longrightarrow} M_{k}
$$

be a partial hierarchy for $M$ and let $N=\mathcal{N}\left(\partial M \cup S_{1} \cup \cdots \cup S_{k-1}\right)$ be a regular neighbourhood of the union of all the surfaces in the hierarchy. Then:

1. The surface $\partial N-\partial M$ is incompressible in $N$.

2. Compressing the surface $\partial N-\partial M$ into the 3-manifold $M-\operatorname{int} N$ as much as possible gives a disjoint union of closed connected separating surfaces, each of which is either incompressible in $M$ or is a 2-sphere bounding a 3-ball in $M$.

Proof. Let's start by observing that the (partial) hierarchy for $M$ from the lemma gives a partial hierarchy for $N$, where the manifold we end up with after cutting along surfaces $S_{1}, \ldots, S_{k-1}$ is homeomorphic to $(\partial N-\partial M) \times I$. Now we proceed by induction on $k$. For $k=2$ we have a single incompressible surface $S_{1}$. We can assume that a compression disc $D$ for $\partial N-\partial M$ (in $N$ ) intersects $S_{1}$ in simple closed curves only and that it is disjoint from $\partial M$. Every component of $S_{1} \cap D$ is homotopically trivial and hence bounds a disc in $S_{1}$ (since $S_{1}$ is incompressible). The innermost such curve in $S_{1}$ bounds a disc $D^{\prime}$ in $S_{1}$ disjoint from $D$. By cutting 
$D$ along $\partial D^{\prime}$ and gluing in a parallel copy of $D^{\prime}$ we obtain a new compression disc (again denoted by $D$ ), such that the number of components of the intersection $D \cap S_{1}$ is reduced by at least one. By repeating this procedure we can make $D$ disjoint from $S_{1}$. This implies that $D$ is in fact a compression disc for the surface $\partial N-\partial M$ in the product $(\partial N-\partial M) \times I$. So its boundary has to bound a disc in $(\partial N-\partial M)$. The inductive step is proved in the similar fashion by first making the compression disc disjoint from the surface $S_{1}$, and then applying the induction hypothesis to the partial hierarchy in $M_{S_{1}}$. This proves part 1 .

The closed surface $\partial N-\partial M$ is separating in $M$ and can only compress into $M-$ int $N$. Furthermore, these new compression discs can be viewed as the continuation of the existing partial hierarchy. So by applying the first part of the lemma to this extended hierarchy, we see that each component of the compressed surface is either incompressible both in the regular neighbourhood of the extended hierarchy and in its complement, or it is a 2-sphere. In both cases part 2 of the lemma follows.

The distinguished two-dimensional polyhedron lying inside of the hyperbolic pieces of $M$ can now be topologically, but not yet algorithmically, described as follows. It will consist of the union of surfaces in the distinguished hierarchy which is given by the following three-step procedure. We should note here that there are many different choices for the surfaces in the hierarchy we are about to describe. The term "distinguished" simply means that we can make one choice and stick with it in any triangulation of our 3-manifold $M$.

Step 1. Let $S_{1}$ be the first surface in the hierarchy. Its components will be defined recursively. We start by adjoining all canonical tori and annuli from the JSJ-system of $M$. In other words at this point $S_{1}$ consists of the surfaces $\partial \Sigma-\partial M$, where $\Sigma$ is the characteristic submanifold of $M$. We will adjoin new closed connected surfaces to $S_{1}$ in the components of the JSJ-decomposition of $M$ which are not contained in $\Sigma$ and are neither homeomorphic to a solid torus nor to a (torus) $\times I$. Boundary of such a piece might or might not be incompressible in $M$. For each incompressible boundary component of such a component, we add a parallel copy of it to our surface $S_{1}$ (this makes sure that all the complementary pieces we need to work on in Step 2 are pure). In each component of the complement $M-$ int $\Sigma$ defined above, the rest of the surface $S_{1}$ will consist of disjoint closed connected orientable incompressible surfaces. If two components of $S_{1}$ are parallel, then no other connected subsurface in $S_{1}$ will be parallel to them. They are defined by the following recursion.

1a. If the complementary piece of the surface we have defined so far contains no canonical annuli, then we proceed by looking for a closed, injective surface that is not boundary parallel (in that piece), and that has the largest Euler characteristic (smallest genus) out of all such surfaces in our piece. If a surface like 
that does not exist, $S_{1}$ gets no new components in the piece we are studying. If it does exist, we adjoin to $S_{1}$ a boundary of its regular neighbourhood in our piece.

1b. If, on the other hand, our submanifold contains a canonical annulus, we look at the union of all canonical annuli with the boundary of the piece. Compressing the boundary of the regular neighbourhood of that two-dimensional polyhedron as far as we can, gives a disjoint union of closed separating orientable surfaces. If this union contains a surface that is neither homeomorphic to $S^{2}$, nor parallel to any of the boundary components of the piece, then we adjoin to $S_{1}$ a boundary of a regular neighbourhood of a closed injective surface (in our piece), which is not boundary parallel, and that has the largest negative Euler characteristic among all such surfaces. If there aren't any such surfaces, we do nothing.

The union of all the surfaces in $M$ obtained in this way, defines the surface $S_{1}$ in our hierarchy.

Step 2. Let's look at a piece of the cut-open manifold $M_{S_{1}}$ that is not homeomorphic to an $I$-bundle over a surface and is not contained in the characteristic submanifold of $M$. Such a piece either contains a canonical annulus or it doesn't. If it does, take the surface $S_{2}$ in that piece to be a union of two parallel copies of each canonical annulus in it. If it doesn't, let $S_{2}$ be the horizontal boundary of a regular neighbourhood of a connected bounded two-sided incompressible surface with the largest Euler characteristic.

Step 3. The pieces of $M$ after the first two steps are either homeomorphic to compression bodies, whose negative boundary $\partial_{-}$can be empty (the case of handlebodies) or disconnected, or to $I$-bundles over (perhaps nonorientable) closed surfaces. All the subsequent surfaces in the distinguished hierarchy are going to be annuli and discs and are going to be defined recursively using boundary patterns on the pieces.

This construction both raises a number of questions and also requires some explanation. The recursion defining the surfaces $S_{1}$ must stop by the Kneser-Haken finiteness theorem (see [Jaco 1980] and [Hempel 1976]). Moreover it follows that $M$ can contain at most $8 t+\beta_{1}(M ; \mathbb{Z})+\beta_{1}\left(M ; \mathbb{Z}_{2}\right)$ closed connected incompressible surfaces that are nonparallel, where $t$ is the number of tetrahedra in $T$. Therefore the surface $S_{1}$ can contain at most $2 \cdot 20 t$ components, since the Betti numbers $\beta_{1}(M ; \mathbb{Z})$ and $\beta_{1}\left(M ; \mathbb{Z}_{2}\right)$ are smaller than $6 t$.

It is clear from the definition of $S_{1}$ that all components of the cut-open manifold obtained at each stage of the recursion defining $S_{1}$, which are disjoint from the characteristic submanifold $\Sigma$, are atoroidal. So the set of all surfaces in the 
JSJ-system of each such 3-dimensional piece is either empty or it contains only annuli. All components of the 3-manifold $M_{S_{1}}$ have nonempty boundary. It is well known (see [Hempel 1976]) that a bounded compact irreducible 3-manifold that is not a 3-ball contains an orientable (and hence two-sided) nonseparating properly embedded incompressible surface with boundary. So even in the components of $M_{S_{1}}$ that contain no canonical annuli, we can still find and fix one such surface with the largest Euler characteristic. Therefore we can carry out Step 2 as well.

In order to determine what the pieces of the complement look like topologically after Step 2 we need the following definition. A compression body is a connected orientable 3-manifold obtained from a (possibly disconnected) closed orientable surface $S$ by attaching 1-handles to the surface $S \times\{1\}$ in the boundary of the product $S \times I$. The negative boundary $\partial_{-}$of the compression body is precisely the incompressible part of its boundary, which equals $S \times\{0\}$. The rest of the boundary is called positive and is denoted by $\partial_{+}$. It is convenient to regard handlebodies as compression bodies with empty negative boundary $\partial_{-}$.

We now have to examine the boundary of the regular neighbourhood $N=$ $\mathcal{N}\left(\partial M \cup S_{1} \cup S_{2}\right)$. The boundary of $N$ is a disjoint union of closed surfaces, some of which are parallel to the components of the surface $\partial M \cup S_{1}$ and some of which are not. The former are incompressible in $M$. The latter are incompressible only in $N$. Lemma 4.1 also tells us that these surfaces either compress all the way, and therefore bound handlebodies, or they become closed incompressible surfaces in $M$ after a few compressions. The second alternative implies that they have to be parallel (in $M$ ) to components of $\partial M \cup S_{1}$. This follows from the construction of $S_{1}$, since all closed orientable incompressible surfaces in any component of the 3-manifold $M_{S_{1}}$ have to be boundary parallel. Putting all this together we can conclude that each piece of the cut-open manifold $M-$ int $N$ is homeomorphic to a handlebody, a compression body with a possibly disconnected negative boundary $\partial_{-}$, or an $I$-bundle over a (possibly nonorientable) closed incompressible surface.

All surfaces in Step 3 of our hierarchy will be annuli and discs. The topological information coming from the manifold $M$ will be reflected in the boundary patterns (as described in Section 2B) on the boundaries of the pieces.

There is a very natural way of obtaining boundary pattern from any partial hierarchy of $M$. Let

$$
M_{1} \stackrel{S_{1}}{\longrightarrow} M_{2} \stackrel{S_{2}}{\longrightarrow} \cdots \stackrel{S_{k-1}}{\longrightarrow} M_{k}
$$

be such a hierarchy and let $K$ denote a two-dimensional polyhedron that is a union of $\partial M$ with all the surfaces from this hierarchy. The singular locus $S(K)$ of the polyhedron $K$ consists of all the points in $K$ that do not have neighbourhoods homeomorphic to discs (i.e., those points whose links in $K$ are not circles). Let $Q$ 
be a closure (in $M$ ) of a component of $M-K$. In our setting $Q$ is always an embedded submanifold of $M$. It also inherits the boundary pattern from the hierarchy in the following way: $P=\partial Q \cap S(K)$. Note that $P$ consists of trivalent graphs and disjoint simple closed curves, and that the surface $\partial Q-P$ is by definition incompressible in $Q$.

Now let $Q$ be a complementary piece of the distinguished hierarchy after Step 2. The pattern on $\partial Q$ consists of a (possibly empty) collection of disjoint simple closed curves. All the subsequent surfaces in the distinguished hierarchy that are contained in $Q$ are going to be annuli and discs. They will be defined using the information coming from the pattern. We will adjoin them in such a way that the closures of the complementary components at any step of the hierarchy are embedded submanifolds.

Before describing the next step in the distinguished hierarchy, we need to impose some pattern on the pure pieces of the form (torus) $\times I$ that are not contained in the characteristic submanifold $\Sigma$. Such submanifolds arise from canonical tori in $M$ that are separating Seifert fibred pieces with nonmatching fibrations. So on each boundary component of (torus) $\times I$, we take our pattern to be the regular fibre of the Seifert fibration on that side. Since the fibrations do no match (if they did, the defining torus of the piece would not be canonical), this pattern admits no nontrivial $P$-essential annuli in our piece.

The third step in the construction of the distinguished hierarchy will be carried out in three substeps:

3a. Fix an ordering of the 3-manifold components of the complement $M-\left(S_{1} \cup S_{2}\right)$ that are not contained in the characteristic submanifold $\Sigma$. Go through all the pieces one by one, respecting this ordering, and each time add a disjoint maximal collection of $P$-canonical annuli that exist in that piece. Every time new $P$-canonical annuli are adjoined, the pattern on the boundaries of the neighbouring pieces acquires some additional simple closed curves. Also, if a $P$-canonical annulus does not separate the piece it lies in, we add two copies of it. When we are done with all the components of $M-\left(S_{1} \cup S_{2}\right)$, we fix a new ordering on the pieces of $M-\left(S_{1} \cup S_{2} \cup S\right)$, where $S$ equals the union of all $P$-canonical annuli we have added in so far. We repeat the procedure above on this new ordering. We iterate the whole process until we reach an ordering of the complementary components with boundary patterns admitting no $P$-canonical annuli in any of the pieces.

3b. At this step of the hierarchy all the complementary pieces that contain nontrivial $P$-essential annuli are $I$-bundles with pure horizontal boundaries (see Theorem 2.1). In this step we simplify proper compression bodies and $I$ bundles over closed surfaces down to handlebodies by inserting the spanning 
annuli. First we fix a complementary piece that is a compression body. We adjoin each trivial $P$-essential annulus in our piece with the following property: the annulus in the boundary of the piece that it is parallel to contains precisely two pure subannuli and no simple closed curve components of the pattern (this technical requirement ensures that the adjoined trivial $P$-essential annulus is uniquely determined by the existing pattern on the boundary of our piece). The topology of our complementary piece remains unchanged after we have added all such trivial $P$-essential annuli. We need these annuli so that normal surface theory can be applied to decompose the piece. Now we adjoin an incompressible spanning annulus that has minimal intersection with the pattern. Spanning in the context of compression bodies means that the bounding circles of the annulus lie in distinct boundary components of the compression body. If the chosen spanning annulus is not separating, we take two parallel copies of it. We repeat this procedure for each component of the negative boundary $\partial_{-}$. We now run $3 \mathrm{a}$ again. If there are compression bodies left we repeat $3 \mathrm{~b}$ on one of them. Otherwise we do $3 \mathrm{~b}$ on an $I$-bundle over a closed surface. Once all complementary pieces are handlebodies we move to the next step.

3c. Now all complementary pieces are handlebodies and none of them contains a $P$-canonical annulus. Fix a complementary piece that contains no nontrivial $P$-essential annuli and which is neither a 3-ball nor a solid torus with a pure injective annulus in its boundary. If no such piece exists we stop. Otherwise we adjoin all trivial $P$-essential annuli in our piece that have the same property as the ones described in $3 \mathrm{~b}$. We adjoin them using the same method as in $3 b$. Then we choose a compression disc in our piece having minimal intersection number with the boundary pattern among all compression discs in our handlebody. If it is not separating, we take two copies of it. Notice that the compressed handlebody, which is not necessarily connected, satisfies the same conditions as the original piece we have just compressed. So we carry on with the compressions until the original piece becomes a union of 3-balls. Now we run $3 \mathrm{a}$ again. The situation now is precisely as it was at the beginning of $3 \mathrm{c}$, so we can repeat it.

The description of Step 3 requires some explanation. Step 3a is there to eliminate the nontrivial $P$-essential annuli in the complementary pieces. This is crucial for the algorithmic construction of the surfaces in the hierarchy, because such annuli make normal surface theory impossible to apply. In Step 3b we make sure that all complementary pieces are handlebodies. Step $3 \mathrm{c}$ is there to compress these handlebodies down to 3-balls with the exception of solid tori which contain a pure annulus in their boundaries. 
If no complementary piece after Step 3a is homeomorphic to a proper compression body or to an I-bundle over a closed surface, we do nothing in $3 \mathrm{~b}$. On the other hand, if such pieces exist, we need to make sure that they contain no nontrivial $P$-essential annuli, so that we can use normal surface theory later on to construct the vertical annuli.

Lemma 4.2. A compression body from Step $3 b$ in the hierarchy can contain no nontrivial P-essential annuli. When there are no more compression bodies left, any I-bundle over a closed surface from $3 b$ has the same property.

Proof. No piece in 3 b contains a $P$-canonical annulus by 3 a. So a compression body containing a nontrivial $P$-essential annulus would have to be homeomorphic to either an atoroidal Seifert fibred space which is not a solid torus or to an $I$ bundle. This follows directly from Theorem 2.1, which can be applied since all the complementary pieces in Step 3 are atoroidal.

The first possibility cannot occur since all such Seifert fibred spaces have incompressible boundary (see Figure 3 in [Mijatović 2004]). Also a proper compression body is not homeomorphic to an $I$-bundle over a compact surface because it has a compressible boundary component and at least one incompressible one. Since both possibilities lead to contradiction, no compression body can contain a nontrivial $P$ essential annulus after $3 \mathrm{a}$. Notice also that the complement of the spanning annulus in a compression body is again a compression body whose negative boundary has fewer components than that of the original piece.

If there existed an $I$-bundle over a closed surface that contained a nontrivial $P$-essential annulus then, by Theorem 2.1, it would have to be homeomorphic to an $I$-bundle over a compact surface with pattern lying in the annuli that fibre over the boundary circles of the base surface. So our piece is actually pure because any $I$-bundle over a bounded surface has compressible boundary. This means that the boundary of our $I$-bundle is contained in the surface $S_{1}$.

Our piece can in general be adjacent to an $I$-bundle, a compression body or a handlebody.

The first possibility would make $M$ into a closed atoroidal surface bundle over $S^{1}$ or semibundle over $I$, since the boundary of our $I$-bundle is both pure and contained in $S_{1}$ (by definition, $S_{1}$ does not have more than two parallel copies of any component). This contradicts our initial hypothesis on $M$.

The second possibility with the compression body cannot take place, because Step $3 \mathrm{~b}$ does not touch $I$-bundles while compression bodies are still around. But the adjacent piece also cannot be a handlebody since all components of $S_{1}$ are incompressible in $M$. All this implies that, when there are no more compression bodies in the complement of the hierarchy, each $I$-bundle over a closed surface contains no nontrivial $P$-essential annuli. 
After we have adjoined the spanning annuli in all the compression bodies and $I$-bundles over closed surfaces (i.e., after we have completed Step 3b), every complementary region of the hierarchy becomes a handlebody.

In Steps $3 b$ and $3 c$ we also have to adjoin certain trivial $P$-essential annuli to the complementary pieces. If the pattern on the boundary of our piece consists of simple closed curves only, we do not need to adjoin such annuli. Otherwise we add in the ones that are parallel to the annuli in the boundary of the piece which contain no simple closed curves of the pattern and do contain precisely two pure subannuli. This rule makes sure that no two of the trivial $P$-essential annuli we add are topologically parallel (that is, parallel disregarding the pattern). This process creates more of the solid tori, in the complement of the hierarchy, which contain injective pure annuli in their boundaries. But it does not change the topology of the complementary piece. The reason we have to adjoin the trivial $P$-essential annuli before applying normal surface theory is that we need to make sure that any surface $F$ we are trying to find, be it a disc or an annulus, is isotopic, by an isotopy that is invariant on the pattern, to a surface obtained from $F$ by twisting along a trivial $P$-essential annulus. It is clear that after we adjoin the trivial annuli this can be achieved.

Now that we have fully described the distinguished hierarchy, we need to show that Step 3 (i.e., 3a and hence most other substeps) does not run forever and that, when it terminates, the complementary pieces of the hierarchy we get are solid tori with pure annuli in their boundaries and 3-balls. To do that we have to understand how incompressible (but not $\partial$-incompressible) annuli lie in handlebodies and compression bodies. In fact we can concentrate only on the annuli that are not $\partial$-parallel in the piece we adjoin them to, because the number of the boundary parallel ones is easily controlled by the complexity of the pattern.

It follows directly from the definition that a $P$-canonical annulus is not boundary parallel in the complementary piece we adjoin it to. Let's start by looking at a handlebody component $H$ of $M-$ int $\mathcal{N}\left(S_{1} \cup S_{2}\right)$. The process described above creates a sequence of pure incompressible properly embedded annuli in $H$. Moreover no two annuli can be parallel, because that would make one of them $\partial$-parallel in a submanifold of $H$. So what we have is a disjoint collection of properly embedded nonparallel annuli in the handlebody $H$ (here we are only taking into account one of the two parallel copies of $P$-canonical annuli that we sometimes had to adjoin, because the annulus was not separating), none of which is $\partial$-parallel.

There are at most $6 g$ such annuli, where $g$ is the genus of handlebody $H$. To see this, suppose $\mathscr{A}$ is the union of at least $6 g$ such annuli in $H$. We can then perform a sequence of $\partial$-compressions to $\mathscr{A}$ until we end up with a $\partial$-incompressible surface. Since the only such surfaces in handlebodies are discs, we get a collection of at least $6 g$ disjoint discs in $H$. Now, each annulus in $\mathscr{A}$ is compressed only once and 
the disc it yields, after the $\partial$-compression, is a compression disc for $\partial H$. This is because none of the components of $\mathscr{A}$ are $\partial$-parallel. But there are at most $3 g-3$ disjoint nonparallel compression discs in a handlebody of genus $g$. So at least three of our discs are parallel. It is easy to see that, if we reconstruct the annuli corresponding to these parallel discs by reversing the $\partial$-compressions, at least two of the annuli are going to be parallel in $H$.

The same proof tells us that in a compression body $H$ we cannot have more than $6 g\left(\partial_{+} H\right)$ incompressible $\partial$-compressible annuli that are neither $\partial$-parallel nor parallel to each other (a compression body contains at most $3 g\left(\partial_{+} H\right)-3$ disjoint nonparallel compression disc). On the other hand, compression bodies do contain incompressible $\partial$-incompressible annuli. We can always choose the handle structure of the compression body $H$ so that our family of annuli is vertical in the product structure of the complement of the 1-handles. This can be seen as follows. Choose a family of compression discs in $H$ which cuts it down to $\left(\partial_{-} H\right) \times I$ and which intersects our annuli in the minimal number of arcs. Since all the annuli are $\partial$-incompressible, all the arcs of intersection must be inessential. Consider an outermost arc separating off a disc $D$ in one of the annuli. The arc lies in one of the compression discs and divides it into subdiscs $D^{\prime}$ and $D^{\prime \prime}$. Remove $D^{\prime}$, say, from the compression disc and replace it with $D$. By choosing $D^{\prime}$ appropriately we may ensure that the family of compression discs we obtain after this operation still cuts $H$ down to $\left(\partial_{-} H\right) \times I$. Clearly this family of discs intersects our annuli in fewer arcs, which is a contradiction.

This implies that there are no more than

$$
\left(3 g\left(\partial_{-} H\right)-3\right)+2\left(g\left(\partial_{+} H\right)-g\left(\partial_{-} H\right)\right)
$$

nonparallel incompressible $\partial$-incompressible annuli in $H$. The first summand bounds the number of disjoint nonparallel simple closed curves in the surface $\partial_{-} H$. The second summand accounts for the fact that two vertical annuli parallel in $\partial_{-} H \times I$ need not be parallel in $H$. Since each 1-handle of $H$ produces two discs in the boundary of $\left(\partial_{-} H\right) \times I$, we get the bound. Putting all these numbers together we can conclude that each compression body can contain no more than $9 g(\partial H)$ disjoint nonparallel incompressible annuli that are not $\partial$-parallel.

We are now going bound the genus of any closed surface that bounds a compression body or a handlebody from $M-\operatorname{int} \mathcal{N}\left(S_{1} \cup S_{2}\right)$ (see Corollary 4.5). This bound implies that there are only finitely many compression discs in $3 \mathrm{c}$. The number of annuli in Steps 3a, 3b and 3c, which are not boundary parallel, is, by the discussion in the previous paragraphs, controlled by the genus of these surfaces. Each trivial $P$-essential annulus featured in Steps $3 \mathrm{~b}$ and $3 \mathrm{c}$ is always parallel to an annulus in the boundary of the piece which contains some pattern. This pattern is such that it must contain at least one simple closed curve that is embedded in it and that is 
a boundary component of some surface in the hierarchy that was adjoined to the neighbouring piece at some earlier instance (notice that this simple closed curve must contain at least one vertex of the pattern). Corollary 5.8 in [Tollefson and Wang 1996] gives us control over the negative Euler characteristic of nonannular components of $S_{2}$. The number of boundary components of annular pieces of $S_{2}$ is bounded by $20 \cdot 18 t$, where $t$ is the number of tetrahedra in $M$. This follows from the Kneser-Haken finiteness theorem and the fact that all canonical annuli lie in the complement of the amalgams (see the proof of Corollary 4.5). So we have complete control over the number of boundary components of the surface $S_{2}$. Also, when we adjoin trivial $P$-essential annuli, the pattern that they generate cannot contribute to the birth of new trivial $P$-essential annuli. Therefore, since we can bound the number of $P$-canonical annuli, we can also bound the number of trivial $P$-essential annuli that come out of $3 \mathrm{~b}$ and $3 \mathrm{c}$. All this implies that the procedure described by Step 3 in the construction of the distinguished hierarchy must terminate.

The rest of this section is devoted to showing that all the embedded submanifolds in the complement of the whole distinguished hierarchy, after we have carried out Step 3, are indeed 3-balls and tori that contain pure annuli in their boundaries. Since Step 3a cannot run forever under any circumstances, we can assume that Steps $3 b$ and $3 \mathrm{c}$ have been implemented as well. This means that all the complementary pieces are either handlebodies not containing $P$-canonical annuli, or 3 -balls. The only pieces we need to worry about are the handlebodies which contain nontrivial $P$-essential annuli (the ones that do not have already been compressed all the way in Step 3c). We will now show that such handlebodies support a natural $I$-bundle structure with pure horizontal boundaries. Moreover we will see that they are stacked one on top of the other along their horizontal boundaries, forming a surface bundle or semibundle.

Assume that, after Step 3 of the hierarchy has been done, there is a complementary piece $Q$ containing a nontrivial $P$-essential annulus. By Theorem 2.1, it has to be an $I$-bundle over a bounded surface of negative Euler characteristic. The pattern is contained in the vertical boundary and the horizontal boundary is pure.

Now we look at the complementary piece $Q^{\prime}$ which is on the other side of the horizontal boundary of $Q$. We know that $Q^{\prime}$ is a (possibly trivial) handlebody containing no $P$-canonical annuli (by 3 a). We will now show that it must contain a nontrivial $P$-essential annulus. Once we establish that, the $I$-bundle structure of $Q^{\prime}$ will follow from Theorem 2.1.

The horizontal boundary of $Q$ is incompressible in $Q^{\prime}$, by the definition of a hierarchy. $Q^{\prime}$ is therefore not a 3-ball. Furthermore $Q^{\prime}$ must contain a nontrivial $P$-essential annulus, because if it didn't, its boundary would have been compressed down to a 2 -sphere by 3 c. Since $Q^{\prime}$ doesn't contain any $P$-canonical annuli, we 
can apply Theorem 2.1 to conclude that $Q^{\prime}$ is an $I$-bundle over a bounded surface as well. Moreover the horizontal boundary of that $I$-bundle is pure. It is either connected or it has two components, depending on whether the base surface is nonorientable or not. In the first case the horizontal boundary of $Q^{\prime}$ has to coincide with a component of the horizontal boundary of $Q$. In the second case a component of the horizontal boundary of $Q^{\prime}$ matches a component of the horizontal boundary of $Q$. If $Q$ and its neighbour meet along all of their horizontal boundaries, their union is an embedded submanifold $N$ in $M$ that is a surface bundle or semibundle with the fibre a bounded surface. If not, we can extend the $I$-bundle structure over the union $Q \cup Q^{\prime}$. We can repeat the argument for the enlarged $I$-bundle and the pieces adjacent to it. After finitely many repetitions we must arrive at the surface bundle or semibundle situation described above, since we are in the complement of the characteristic submanifold $\Sigma$. (If the horizontal boundary of the extended $I$-bundle hits $\partial M$, it is not hard to see that the annuli in vertical boundary of the $I$-bundle are incompressible and $\partial$-incompressible in $M$, making our $I$-bundle a subset of $\Sigma$.) The following lemma will give the final contradiction with our initial hypothesis on the 3-manifold $M$.

Lemma 4.3. The submanifold $N$ constructed above is a bounded strongly simple piece of the JSJ-decomposition of the 3-manifold $M$. In particular, $N$ is atoroidal and an-annular. For the surfaces $S_{1}$ and $S_{2}$ obtained after the first and second steps in the construction of the distinguished hierarchy, we have $\partial N-\partial M$ equal to $N \cap S_{1}$ and consisting of canonical tori from $M$; and we have $N \cap S_{2}$ equal to either a union of two parallel components of $S_{2}$ (if $N$ is a surface bundle over $S^{1}$ ) or a single component of $S_{2}$ (if $N$ is a surface semibundle over I).

Proof. Since $M$ is fibre-free and the submanifold $N$ lies in the complement of $\Sigma$, the surface fibre of $N$ cannot be closed. In other words, the boundary of $N$ cannot be empty. First we need to show that the components of $\partial N$ are incompressible tori in $M$. Let $S$ be one such torus. Assume that it is compressible. Then it either bounds a solid torus in $M$ or is contained in a 3-ball in $M$. But $S$ contains a boundary circle of the fibre of $N$. Since this surface injects in $M$, the torus $S$ cannot be contained in a 3-ball, nor can it bound a solid torus with the slope of the meridian disc equaling the slope of the fibre of $N$.

Let $F$ be a connected surface in the distinguished hierarchy that contains one of the fibres of the surface bundle $N$. It is clear that $F$ has to be either a component of the closed surface $S_{1}$ or a nonannular component of the bounded surface $S_{2}$. In both cases we are going to consider how the surface $F$ interacts with the solid torus bounded by $S$ (we can assume without loss of generality that the solid torus is disjoint from int $N$ ). We have two possibilities. The surface $F$ is either disjoint from its interior, or it intersects it. In the former case it has to contain another 
surface fibre of $N$. This leads directly into contradiction because it renders $F$ compressible in $N$ and hence in $M$. In the latter case the subsurface of $F$ that is contained in the solid torus has to be injective in the solid torus. This follows from the injectivity of $F$ and the fact that the intersection $F \cap S$ has to inject into $M$ as well, since it is part of the pattern. So $F$ consists of annuli in the solid torus bounded by $S$ (Möbius bands are ruled out by orientability of $F$ ). This means that again $F$ has to contain at least two surface fibres of $N$. Take an annulus from $F$ that is outermost in the solid torus bounded by $S$. The two fibres of $N$ that are contained in $F$ and lie one on each side of this annulus can be used to show that in this case $F$ has to be compressible as well. This proves that the surface $\partial N$ is incompressible in $M$.

Since the submanifold $N$ is disjoint from the characteristic submanifold $\Sigma$, each boundary torus from $\partial N$ has to be parallel either to a canonical torus in $M$ or to a toral boundary component of $M$. Therefore $N$ has to be a strongly simple piece of the JSJ-decomposition of $M$. If an essential annulus in $(N, \partial N)$ is canonical then, by Proposition 4.1 from [Neumann and Swarup 1997], it has to be a matched annulus, making $N$ into a Seifert fibred space. If $(N, \partial N)$ contains no canonical annulus and yet contains an essential one, then it is again Seifert fibred by Proposition 3.2 in [Neumann and Swarup 1997]. This implies that $N$ is in fact an-annular.

It follows from the construction of $N$ that any surface from the intersection $N \cap S_{1}$ will either contain the whole boundary component of $N$ or it will contain a surface fibre of $N$. The latter possibility cannot occur because any component of $S_{1}$ that is not contained in $\partial N$ has to be disjoint from it and can therefore not carry a fibre of $N$. The intersection $N \cap S_{2}$ consists of components of $S_{2}$ which are therefore surface fibres of $N$. But since $S_{2}$ contains at most two parallel copies of an incompressible $\partial$-incompressible surface the lemma follows.

4A. Topological complexity. There are two kinds of complexities of the surfaces in the distinguished hierarchy we need to consider. First there is the normal complexity, i.e. the number of normal pieces a minimal weight representative in the isotopy class of the surfaces consist of. We will deal with it in Section 4B. Second there is the topological complexity of the surfaces in the hierarchy, that is defined in terms of their components in the following way. To each component we assign its negative Euler characteristic and then define the complexity to be the sum over all of its components. Topological complexity of the surfaces $S_{1}$ and $S_{2}$ will, together with the Kneser-Haken finiteness theorem, determine the number of connected surfaces we needed to cut along. Bounding it therefore provides a crucial step in the actual construction of the distinguished hierarchy. Since there are no 2-spheres, discs or projective planes in the first two steps of the hierarchy, our topological complexity coincides with the Thurston complexity as defined in [Thurston 1986]. 
Lemma 4.4. Let $M$ be a triangulated irreducible 3-manifold with (possibly empty) incompressible boundary that is different from $\mathbb{R} P^{3}$. Assume further that the manifold $M$ is atoroidal and that it is not homeomorphic to an I-bundle. Then any closed injective surface in $M$ that is not boundary parallel and that has the smallest topological complexity among all such surfaces is ambient isotopic to a fundamental surface.

Proof. The general strategy of the proof is to apply a version of Theorem 2.3 to an injective surface of minimal complexity and thus conclude that it is fundamental. But first we need to collect some facts about $M$ and about normal surfaces in it.

Claim. $M$ cannot contain a projective plane or an injective Klein bottle.

Proof. A boundary of a regular neighbourhood of an injective Klein bottle is an incompressible torus. But all such tori in $M$ are boundary parallel, making $M$ into an $I$-bundle. Since $M$ is irreducible and not homeomorphic to $\mathbb{R} P^{3}$, it cannot contain an embedded projective plane.

The next result is well known.

Sublemma. If $F$ is a connected normal surface which is a sum $F=F_{1}+F_{2}$, then it can be expressed as another sum $F=F_{1}^{\prime}+F_{2}^{\prime}$, where both normal surfaces $F_{1}^{\prime}$ and $F_{2}^{\prime}$ are connected and no component of the intersection $F_{1}^{\prime} \cap F_{2}^{\prime}$ separates both surfaces. Furthermore we can assume that the sum $F=F_{1}^{\prime}+F_{2}^{\prime}$ is in reduced form. Proof. The proof is by making regular alterations along certain arcs and circles in $F_{1} \cap F_{2}$. The procedure, we are just about to describe, must terminate because there is only finitely many components in $F_{1} \cap F_{2}$. If, say, $F_{1}$ is not connected, fix one of its components and do all regular alterations along curves in the intersection between all other components and $F_{2}$. Name the component we fixed (and didn't touch) $F_{1}$ again, and call the new normal surface $F_{2}$. Notice that the number of components of $F_{1} \cap F_{2}$ is smaller than what it was before. Now, if $F_{2}$ is not connected, we can reiterate the above procedure with $F_{2}$ taking the place of $F_{1}$ and vice versa. The repetition of these steps must eventually terminate with both $F_{1}$ and $F_{2}$ being connected.

Assume now that there exists a component of $F_{1} \cap F_{2}$ that separates both surfaces into

$$
F_{i}=F_{i}^{(1)} \cup F_{i}^{(2)} \quad \text { for } i=1,2 .
$$

This cannot be the only component of $F_{1} \cap F_{2}$ because the surface $F$ is connected. Choose notation so that the regular alteration along this component pastes $F_{1}^{(j)}$ with $F_{2}^{(j)}$ for $j=1,2$. Doing regular alterations along the components of $F_{1}^{(j)} \cap F_{2}^{(j)}$, for $j=1,2$, produces two normal surfaces, called $F_{1}$ and $F_{2}$ again, with fewer components in their intersection and with the property $F=F_{1}+F_{2}$. If either of them is not connected, we repeat the procedure for making them connected. 
Alternating between the above two processes will eventually produce surfaces $F_{1}$ and $F_{2}$ that are connected, their normal sum $F_{1}+F_{2}$ equals $F$, and no component of the intersection $F_{1} \cap F_{2}$ is separating in both surfaces.

Now take two normal surfaces $G$ and $H$ that satisfy all these three conditions and their intersection has the smallest number of components among all such pairs of surfaces. We claim that the sum $F=G+H$ is in reduced form. Otherwise we can isotope the surfaces $G$ and $H$ to normal surfaces $G^{\prime}$ and $H^{\prime}$ so that the sum $F=G^{\prime}+H^{\prime}$ is in reduced form. This means that the number of components in $G^{\prime} \cap H^{\prime}$ is strictly smaller than the number of pieces of $G \cap H$. The surfaces $G^{\prime}$ and $H^{\prime}$ are still connected, but the third condition from above must fail. But then we can repeat the procedure described above on $G^{\prime}$ and $H^{\prime}$, possibly reducing the number of components in $G^{\prime} \cap H^{\prime}$ even further, making sure that both summands are connected and that no component of intersection separates both of them. This contradicts the choice of $G$ and $H$ and hence proves the sublemma.

Now we can prove the lemma. Suppose $F$ is a closed injective surface in $M$ of the smallest topological complexity. It is therefore connected and incompressible. We isotope it into normal form so that it is weight minimising. Assume now that $F$ is not fundamental and can therefore be expressed as a sum $F=F_{1}+F_{2}$ and that the conclusion of the sublemma is satisfied. Now we can apply the main theorem of [Bart and Scharlemann 1996] to $F=F_{1}+F_{2}$, without isotoping the summands, because the sum is in reduced form. This gives that the surfaces $F_{1}$ and $F_{2}$ are also injective. Moreover, it follows from Lemma 2.2 that neither of the surfaces $F_{i}$ is a 2-sphere. Since the Euler characteristic is additive over normal sums and $M$ contains no projective planes, we now have that $\chi(F)=\chi\left(F_{1}\right)$ and $\chi\left(F_{2}\right)=0$. Since $F_{2}$ cannot be an injective Klein bottle, by the above claim, it can only be a boundary parallel parallel torus. Since $F_{2}$ is connected, it can contain only one copy of such a torus.

We now have to consider the intersection $F_{2} \cap F_{1}$. None of the simple closed curves from $F_{2} \cap F_{1}$ are homotopically trivial in either of the two surfaces because, by Lemma 2.2, there are no trivial patches. So the space $F_{2} \cap F_{1}$ is a 1-manifold that is homeomorphic to a disjoint union of nontrivial parallel simple closed curves in the torus $F_{2}$. Let $X$ be the (torus) $\times I$ region between $F_{2}$ and the toral boundary component of $M$ that $F_{2}$ is parallel to. Then the components of the surface $F_{1} \cap X$ must be injective in $X$, simply because the patches of $F=F_{1}+F_{2}$ are injective by Lemma 2.2, and $\partial X$ is incompressible in $M$. So, since $F_{1} \cap X$ contains no closed components, it consists of incompressible annuli that are disjoint from the torus $X \cap \partial M$. Each such annulus must be topologically parallel to an annulus in $F_{2}$. Let $B$ be an outermost annular component of $F_{1} \cap X$, lying in the product region $X$, and let $A$ be the annulus in $F_{2}$ that is parallel to $B$. There are three possible 

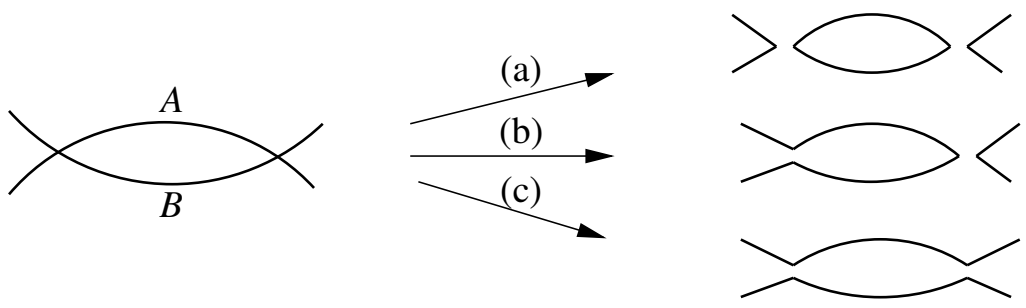

Figure 4. Possible normal alterations.

(essentially different) ways a normal alteration can act on $\partial A$. They are depicted by Figure 4.

They all lead to contradiction. Case (a) produces a disconnected sum. In case (b) we can isotope the union of the patches $A$ and $B$ over the solid torus that they bound, to reduce the weight of $F$. If both $A$ and $B$ had zero weight, then there would exist a normal isotopy that would reduce the number of components in $F_{1} \cap F_{2}$. This contradicts the reduced form assumption. Case (c) contradicts it as well, because the surfaces we obtain after we do the normal alterations along $\partial A$, are isotopic to $F_{1}$ and $F_{2}$, but have fewer components of intersection. This proves the lemma.

Now we are in the position to bound the topological complexity of surfaces $S_{1}$ and $S_{2}$ in the distinguished hierarchy. The next corollary will follow from the construction of the first two surfaces in the hierarchy, from Corollary 5.8 in [Tollefson and Wang 1996] and Lemma 4.4 and also from the bound in Lemma 6.1 of [Hass et al. 1999]. Corollary 4.5 will be used in Section 5 to bound the number of connected surfaces in the distinguished hierarchy.

Corollary 4.5. Let $M$ be a Haken 3-manifold with (possibly empty) boundary. Let $T$ be its triangulation that consists of $t$-simplices. Then the sum of the topological complexities of all closed surfaces in $M$, that bound handlebodies, compression bodies and I-bundles in the complement of the distinguished hierarchy after Step 2 , is bounded above by $2^{150 t}$.

Proof. If $F$ is a normal surface with respect to the triangulation $T$, then the complement of $F$ in $M$ inherits a polyhedral structure from $T$. It is obvious that there are at most 6 polyhedra in any tetrahedron of $T$, lying in the complement of the normal pieces of $F$, that don't inherit a natural product structure of the form (triangle) $\times I$ or (quadrilateral) $\times I$ (see Figure 4 in [Mijatović 2003]). The complementary polyhedra with this product structure are called parallelism regions. We will be interested in the components of the union of all parallelism regions in $M-F$ which are called amalgams. They were precisely defined and studied in [Lackenby 2003, Sections 7 and 8]. Notice that a vertical boundary of an amalgam (i.e. the part 
which is not contained in $F$ ) is a union of annuli. If they are all injective, we say that the amalgam is maximal. It is described in [Lackenby 2003] how every amalgam can be extended uniquely to the maximal one. A key property of maximal amalgams that will be crucial is the following: if a component $M^{\prime}$ of $M-F$, which is different from an $I$-bundle, contains no canonical annuli, then all the maximal amalgams in it are of the form (disc) $\times I$. It is easy to show that under these circumstances the 3-manifold $M^{\prime}$ can be triangulated by less than $18 t$ tetrahedra (left as an exercise).

The negative Euler characteristic of any normal surface is bounded above by four times the number of normal discs that the surface contains. The topological complexity of the surfaces which bound handlebodies, compression bodies and $I$ bundles in the complement of the hierarchy after Step 2, increases each time we add in a closed component of $S_{1}$ and a nonannular component of $S_{2}$. The quantity from the corollary we would like to have an estimate on is smaller than twice the sum of negative Euler characteristics of $S_{1}$ and $S_{2}$. So all we need is:

Claim. The negative Euler characteristic of each component of the surfaces $S_{1}$ and $S_{2}$ is bounded above by $2^{127 t+19}$.

Proof. If a new component of the surface $S_{1}$ is added in a complementary piece which contains no canonical annuli, Lemma 4.4 and the bound from [Hass et al. $1999,6.1]$ imply that $4 \cdot 5 \cdot 18 t\left(7 \cdot 18 t 2^{7 \cdot 18 t}\right)<2^{127 t+19}$ bounds its topological complexity (the bound is 4 times the normal complexity of a fundamental surface in a triangulation with $18 t$ tetrahedra and the factor $5 \cdot 18 t$ bounds the number of distinct types of normal discs contained in the surface). The same bound works for the components of $S_{2}$ because we always adjoin them to the complementary pieces with no canonical annuli. Instead of applying Lemma 4.4 we have to use [Tollefson and Wang 1996, Corollary 5.8].

If we are adding a component of $S_{1}$ to a piece which contains canonical annuli, then the process described in (1b) of the definition of the distinguished hierarchy guarantees that the negative Euler characteristic of the surface we are adding is bounded above by some linear function of $t$. This is because the same is true for the parts of the boundary of the piece which are disjoint from the maximal amalgams contained in the piece. This proves the claim.

We know already that the number of components of $S_{1}$ is bounded above by $2 \cdot 20 t$ (number 2 accounts for the fact that sometimes we use two parallel copies of the same component when defining $S_{1}$ ). This implies that $S_{2}$ has fewer than $80 t$ nonannular components. This is because there is at most two nonannular components of $S_{2}$ in each complementary piece obtained by cutting along $S_{1}$. The Euler characteristic of the surface from the corollary is twice the sum of the Euler 
characteristics of $S_{1}$ and $S_{2}$. So our bound comes from the estimate

$$
2 \cdot(40+80) t 2^{127 t+19}<2^{150 t} \quad \text { for } t \geq 2 .
$$

4B. Normal complexity. We now investigate how many normal discs are needed to construct surfaces from the distinguished hierarchy. For the components of $S_{1}$ and $S_{2}$ with negative Euler characteristic we will use similar techniques to the ones that bounded their genus. For the canonical surfaces we will apply Propositions 2.4 and 2.5. But the nonpure surfaces from Step 3 will require some additional normal surface theory. The estimates of the number of normal pieces will be in terms of the number of tetrahedra in the subdivision of the piece we are adjoining the surfaces to. Not subdividing and using the same triangulation to bound the normal complexity of several levels of the distinguished hierarchy would certainly lead to much better bounds. But passing the information, which is contained in the pattern, down the hierarchy without both subdividing and keeping the pattern itself in the 1 -skeleton proved to be an insuperable task.

Lemma 4.6. Let $M$ be a 3-manifold that either contains a closed injective surface or has a nontrivial JSJ-decomposition. Assume that $M$ has a triangulation $T$ that consists of $t$ tetrahedra. Then the surface $S_{1}$ from the distinguished hierarchy can be isotoped into normal form so that it contains not more than $2^{350 t^{2}}$ normal discs.

Proof. We have already mentioned that Propositions 2.4 and 2.5 together imply that all the surfaces of the JSJ-system of $M$ can be put into normal form so that they consist of not more than $2 \cdot 2^{80 t^{2}}$ normal discs. The rest of the components of $S_{1}$ were obtained by the recursion described in Step 1 of the distinguished hierarchy. In order to bound their normal complexity in terms of the number of tetrahedra in $M$, we can use the same strategy as in Lemma 2.6 in [Mijatović 2003]. There are at most $20 t$ nonparallel connected surfaces in $S_{1}$. The argument from Lemma 2.6 in [Mijatović 2003] gives us that $S_{1}$ contains not more than $2\left(2 \cdot 11 t 2^{11 t}\right)^{20 t}$ copies of a single normal disc type from $T$. The factor 2 in front of the bracket is there to account for the parallel copies of the components of $S_{1}$ that we sometimes have to adjoin. All together there are at most $5 t$ possible distinct normal disc types contained in $S_{1}$. So we have

$$
5 t \cdot 2\left(2 \cdot 11 t \cdot 2^{11 t}\right)^{20 t}+2 \cdot 2^{80 t^{2}}<2^{t+4}\left(2^{12 t+5}\right)^{20 t}+2 \cdot 2^{80 t^{2}}<2^{350 t^{2}} .
$$

The normal complexity of the components of the surface $S_{2}$ contained in any of the pieces of $M-\operatorname{int} \mathcal{N}\left(S_{1}\right)$ is bounded above by $2^{80 s^{2}}$, where $s$ is the number of 3 -simplices needed to triangulate the piece. This follows directly from Proposition 2.5 for canonical annuli and from Corollary 5.8 in [Tollefson and Wang 1996] for bounded surfaces with negative Euler characteristic. We have established already that there are at most $20 t$ regions in the complement of $S_{1}$ where we have to insert 
the components of $S_{2}$. Here $t$ denotes the number of tetrahedra in the triangulation of $M$. So the number of normal discs in the whole of $S_{2}$ is bounded above by

$$
20 t \cdot 2^{80 s^{2}} .
$$

Now we need to bound the normal complexity of the surfaces in Step 3 of the distinguished hierarchy. Proposition 2.5 does that for all $P$-canonical annuli that need to be constructed in the process. In $3 \mathrm{~b}$ and $3 \mathrm{c}$ we might need to adjoin trivial $P$-essential annuli of a certain kind. In the discussion that followed the proof of Proposition 2.5 we showed that such annuli are fundamental, provided our 3manifold contains no nontrivial $P$-essential annuli. All other surfaces that we have to construct have nonempty intersection with the pattern $P$. Let $l(F)$ be the number of points in the intersection $F \cap P$ for any properly embedded surface $F$ which is transverse to the pattern, If the pattern $P$ is contained in the 1-skeleton then the function $l$ is additive over normal summation.

In order to implement Step 3 we need to describe how to construct the nonpure spanning annuli of $3 \mathrm{~b}$ and also how to find compression discs in handlebodies from $3 c$. We will see that in the construction of surfaces with nonempty intersection with the pattern, it is crucial that even the trivial $P$-essential annuli are well behaved in the way that was specified in $3 \mathrm{~b}$ and $3 \mathrm{c}$. The strategy in this case will be slightly different from what we usually do. Instead of proving that our fixed surface, which minimises the intersection with the pattern, is (almost) fundamental, we are first going to find some other fundamental surface $F$ that has the same property as our original one, but is not necessarily isotopic to it. We will then calculate $\imath(F)$. If we express our original surface as a sum of fundamental surfaces, we can use the additivity of $\imath$ to bound the number of summands in the expression.

Lemma 4.7. Let $M$ be an irreducible bounded 3-manifold triangulated by $t$ tetrahedra. Let $P$ be a nonempty boundary pattern in $\partial M$ contained in the 1-skeleton of the triangulation. Assume every $P$-essential annulus in $M$ is parallel to an annulus in the boundary of $M$ that intersects the pattern in a disjoint union of homotopically nontrivial simple closed curves.

(a) Assume further that $M$ is a compression body with nonempty negative boundary $\partial_{-} M$ and that each toral component of $\partial_{-} M$ is pure. If we fix an incompressible annulus in $M$ whose boundary circles lie in distinct components of $\partial M$ and which has minimal intersection with the pattern $P$, among all annuli satisfying these conditions, then we can isotope it into normal form, by an isotopy which is invariant on the pattern, so that it consists of not more than $2^{40 t}$ normal discs. If such an annulus is not separating and we take two parallel copies of it the same bound still holds. 
(b) If $M$ is an I-bundle over a closed surface which is not a torus, then an incompressible d-incompressible annulus A (or its double if necessary) in $M$, which has minimal intersection with the pattern $P$, can be isotoped, by an isotopy that preserves the pattern, into normal form so that it contains less than $2^{40 t}$ normal discs. If the base surface is a torus and if the pattern P consists of two nonhomotopic simple closed curves, one in each boundary component, the same bound holds.

(c) Let $M$ be a handlebody and let $D$ be a compression disc in $M$ which minimises $\imath(D)$ among all compression discs in $M$. Then we can isotope $D$ into normal form, by an isotopy that is invariant on the pattern, so that it contains not more than $2^{40 t}$ normal discs.

The crucial assumption in Lemma 4.7 is the one about $P$-essential annuli in $M$. Put differently it says that $M$ contains no nontrivial $P$-essential annuli and that every trivial one has to be parallel to an annulus in $\partial M$ containing the simplest possible pattern. Before we add in a surface with nonempty intersection with the pattern in Steps 3b and 3c, we always make sure that this hypothesis holds for the piece we are in by first adjoining the trivial $P$-essential annuli that violate this rule. Notice also that the annulus from Lemma 4.7(a) is automatically $\partial$ incompressible since its boundary circles lie in distinct components of $\partial M$. Since it is also incompressible it has to be a "spanning" annulus described in Step 3b.

It is clear that toral boundary components of genuine compression bodies have to be contained in $\partial_{-} M$. The fact that all such tori are pure can be seen as follows. Each such torus is incompressible in the piece we are in and therefore has to be incompressible in the ambient manifold. So it is either contained in the JSJ-system or in the boundary of the ambient manifold. It is clearly pure in the latter case. If it is a canonical torus, then it is there to separate a strongly simple piece from a Seifert fibred piece. It therefore has to be pure again because at this stage we haven't touched the fibred pieces. Notice also the pattern on the (torus) $\times I$ pieces, which are not contained in the characteristic submanifold $\Sigma$, satisfies the assumption from Lemma 4.7(b).

Proof of Lemma 4.7. We will start by proving (a). Let $F$ be an incompressible annulus in a compression body $M$ with one of its boundary circles lying in a given component of $\partial_{-} M$ and the other in $\partial_{+} M$. Assume that $F$ is in normal form and that it has minimal weight among all possible annuli that satisfy the conditions above. Notice that we haven't stipulated anything as far as $l(F)$ is concerned.

Claim 1. The surface $F$ is fundamental.

Assume to the contrary that $F=U+V$. We can apply the Sublemma from the proof of Lemma 4.4. So both $U$ and $V$ are connected, no component of the 
1-manifold $U \cap V$ separates both of them and the sum is in reduced form. Now we will go through all the potential connected surfaces $U$ and $V$ that satisfy the equation $0=\chi(U)+\chi(V)$, showing each time that we get a contradiction.

The techniques that proved Lemma 2.2 imply that there are no disc patches in $F=U+V$ which are disjoint from the boundary of $M$. So neither of the summands is a 2-sphere. Since $M$ does not contain a projective plane we can assume that $U$ is a disc. In that case $V$ is either a punctured torus, a punctured Möbius band or a punctured annulus. There are no simple closed curves in the 1-manifold $U \cap V$, because $U$ contains no disc patches. Therefore the boundaries of the summands must intersect. So the first case cannot occur because it implies that both $\partial U$ and $\partial V$ are contained in a single component of $\partial M$. In the remaining two cases every outermost arc of $U \cap V$ chops off a patch in $U$ which is a genuine $\partial$-compression disc for $V$, since no component of $U \cap V$ separates both surfaces. Let $C$ be one such patch in $U$ which has the smallest weight among all "outermost arc" patches in $U$. If we $\partial$-compress $V$ along $C$ we obtain an anulus which satisfies the same conditions as $F$ (it is incompressible because one of its boundary components coincides with a boundary circle of $F$ ). Its weight is at most that of $F$. But since one of the parallel copies of $C$ (that was inserted in the $\partial$-compression) was pasted in as an irregular normal alteration, there exists a weight reducing isotopy of the annulus. This contradicts the minimal weight assumption on $F$.

Now we have to consider the case $\chi(U)=\chi(V)=0$. If one of the surfaces is closed, then the other surface is an annulus which is lighter than $F$ and has the same properties. Both of them cannot be Möbius bands because then we would have an embedded Möbius band in $M$ with its boundary contained in $\partial_{-} M$. Gluing two such compression bodies along their negative boundaries gives a 3-manifold which can be embedded in $S^{3}$, but which would contain an embedded Klein bottle. This argument also shows that if $V$ is a Möbius band, then $\partial V$ lives in $\partial_{+} M$ and $U$ has to be an annulus. But in this situation $U$ satisfies the defining conditions for $F$ and $w(U)<w(F)$. We get the same contradiction if $V$ is an annulus with both of its boundary circles contained in a single component of $\partial M$. The only case left is when both $U$ and $V$ are annuli with boundaries lying in distinct components of $\partial M$. Since $w(U)<w(F)$ the annulus $U$ has to be compressible in $M$. So it has to bound a cylinder of the form (disc) $\times I$, because its boundary circles lie in distinct components of $\partial M$. There are no arcs in $U \cap V$ which are $\partial$-parallel in either of the annuli. This is because no curve from $U \cap V$ is separating both in $U$ and $V$. Since there are no homotopically trivial simple closed curves in $U \cap V$, the intersection consists of spanning arcs in both annuli. So $V$ intersects the cylinder (disc) $\times I$ in a collection of discs of length four. Now we look at the disc in $\partial M$ which is bounded by a circle from $\partial U$. This disc intersects $V$ in a collection of arcs that decompose it into complementary regions. The situation for an outermost 
such arc is described by Figure 4 (take $A$ to be a subarc in $\partial V$ which is contained in the disc from $\partial M$ ). By an argument analogous to the one following Figure 4, we arrive at a contradiction, which proves the claim.

Each normal disc in $F$ intersects the 1-skeleton of the triangulation in not more than four points. Since $F$ is fundamental and therefore consists of less than

$$
5 t \cdot 7 t \cdot 2^{7 t}
$$

normal discs, the number $l(F)$ is bounded above by $140 t^{2} \cdot 2^{7 t}$. Here we are using the fact that the pattern $P$ lies in the 1-skeleton.

Let $A$ be our spanning annulus which minimises $l(A)$. We can assume that $A$ is in normal form and that it minimises the weight in its ( $P$-invariant) isotopy class.

Claim 2. If $A=X+Y$ and $X$ is a connected surface with nonnegative Euler characteristic, then $l(X)$ is not zero.

We can assume that the sum is in reduced form. Then by Theorem 2.3 and Lemma 2.2 we have that both $X$ and $Y$ are incompressible, $P$-boundary incompressible and that there are no disc patches. So $X$ cannot be a pure disc or a 2-sphere. Projective planes and a Klein bottles do not embed in $M$ so they cannot appear as summands. If $X$ is a torus, then, since it is incompressible, it has to be parallel to a component of $\partial_{-} M$. By the same argument as the one at the end of the proof of Lemma 4.4 we can conclude that the surface $Y$ intersects the parallelism region (between $X$ and the component of $\partial_{-} M$ ) in (circle) $\times I$. Since there is no pattern we are free to isotope the circle from $\partial A$ in this component as much as we like. This makes the summand $X$ redundant and leads into contradiction. If $X$ were a pure $P$-boundary incompressible Möbius band, then $2 X$ would be a $P$-essential annulus in $M$. Such an annulus is $\partial$-parallel by assumption. Since the parallelism region cannot contain a pure Möbius band, the 3-manifold $M$ would have to be a solid torus which is a contradiction.

The last case we need to consider is when $X$ is a $P$-essential annulus. By assumption this annulus has to be trivial. Since it is pure and there are no disc patches in $A$, the components of $X \cap Y$ are either spanning arcs in $X$ or nontrivial simple closed curves in $X$. The latter possibility cannot occur because it would imply that $\partial A=\partial X$ which is a contradiction. Let $R$ be the solid torus between $X$ and an annulus in $\partial M$. The components of $Y \cap R$ are patches in $A$. They all inject into $M$ an hence into $R$. None of them is homeomorphic to a Möbius band because $A$ is orientable. So the surface $Y \cap R$ is either a disjoint union of discs or a disjoint union of annuli. In the latter case each patch from $Y \cap R$ intersect the annulus $X$ in at least two spanning arcs. Using that we can construct a pure $\partial$-compression disc for any patch form $Y \cap R$ (the pattern in $(\partial R)-X$ consists of parallel nontrivial simple closed curves only). This contradicts Lemma 2.2. So all 
the components of the surface $Y \cap R$ are discs. Furthermore if a single patch from $Y \cap R$ intersects $X$ in more than one spanning arc, then, like before, this patch is $P$ boundary compressible. This again contradicts Lemma 2.2. So every patch from $Y \cap R$ intersects each of the components of the pattern in $(\partial R)-X$ in precisely one point. This again follows from the $P$-boundary incompressibility of the patches of $A$. So we can conclude that the patches from $Y \cap R$, which are all topologically discs, are in one-to-one correspondence with the components of $X \cap Y$ and are isotopic by an isotopy which is invariant on the pattern. In particular each arc from $X \cap Y$ chops of a disc in $Y$. There are essentially two ways of performing regular alterations along the arcs in $X \cap Y$ so that the resulting normal surface does not have more components than $Y$. Both of these possibilities yield a surface which is isotopic to $Y$ via an isotopy that is invariant on the pattern. Since $A$ is connected, $Y$ must be an embedded annulus which is isotopic to $A$ and which satisfies the inequality $w(Y)<w(A)$. This contradiction proves claim 2 .

Let's express our annulus $A$ as a sum of fundamental surfaces: $A=k_{1} F_{1}+\cdots+$ $k_{n} F_{n}$. The number of summands in this expression, that have negative Euler characteristic, is bounded above by the number of disc summands. All other surfaces in the sum have nontrivial intersection with the pattern by Claim 2. So by Claim 1 there are less than $280 t^{2} \cdot 2^{7 t}$ summands in the above expression. The number of normal discs in $A$ is thus bounded above by $280 t^{2} \cdot 2^{7 t} \cdot 5 t \cdot 7 t \cdot 2^{7 t}<2^{18 t+14}<2^{40 t-1}$. This proves (a). Part (b) will work in the same way. Claim 1 is easier now because the boundary of our manifold is incompressible which means that we can apply Theorem 2.3. Claim 2 is true as well. The only difference is that when we are dealing with toral boundary, because of our assumption on the pattern, we can perform the isotopy that leads to contradiction and thus shows that there are no toral summands in A. All numerical bounds are the same as in (a).

To prove part (c) of the lemma, we need the following analogue of Claim 2.

Claim 3. Let $D$ be a compression disc in the handlebody $M$ which minimises $\imath(D)$ and which is in normal form and has minimal weight in its ( $P$-invariant) isotopy class. If $D=X+Y$ and $X$ is a connected surface with nonnegative Euler characteristic, then $l(X)$ is nonzero.

The proof is analogous to that of Claim 2 and is left as an exercise. The rest of the proof of part (c) of our lemma is identical to what we did at the end of the proof of (a).

The last type of complementary pieces we need to construct some normal surfaces in are the $I$-bundles that arise as components of the characteristic submanifold $\Sigma$. Our starting point is a triangulated $I$-bundle $M \rightarrow B$ over a possibly nonorientable bounded surface $B$. Let $V$ be the vertical boundary of $M$ with a fixed triangulation. This prescribed simplicial structure on $V$ will arise from the 
distinguished hierarchy on the other side of $V$. The surfaces we are looking for are vertical compression discs that will simplify $M$ to a 3-ball. A vertical disc in $M$ is a one that fibres over an $\operatorname{arc}$ in $B$. Our collection will contain $1-\chi(B)$ vertical compression discs and will be highly nonunique. But we will define it in such a way that any two choices are related by a homeomorphism of $M$ which is an identity on the vertical boundary. In fact this homeomorphism will come from an automorphism of the base surface $B$, which is fixed on the boundary (see Lemma 6.4 in [Mijatović 2004]).

Let $V_{1}$ be the first annulus in an ordering of the components of $V$ and let $D_{1}, \ldots, D_{n}$ be a collection of disjoint vertical discs we want to describe, where $n=1-\chi(B)$. We are assuming that the vertical boundary of each $D_{i}$ (namely $\left.D_{i} \cap V\right)$ intersects the annulus $V_{1}$ and that every other annulus in $V$ intersects precisely one compression disc. Let $g$ be the genus of $B$, which, in case of a nonorientable surface, is a maximal number of $\mathbb{R} P^{2}$ summands it contains when expressed as a connected sum. We also stipulate that if $B$ is orientable (resp. nonorientable) the first $2 g$ (resp. $g$ ) the compression discs have their entire vertical boundaries contained in $V_{1}$. The last requirement is that, even if $B$ is not orientable, the base surface of the $I$-bundle $M-$ int $\mathcal{N}\left(D_{1}\right)$ is orientable.

Now we need to make sure that the vertical boundary of the surface $D_{1} \cup \cdots \cup D_{n}$ interacts in a prescribed way with the triangulation of $V$. Choose a fibre $\lambda$ in each component of $V$ which consists of the smallest number of normal arcs with respect to the given triangulation of $V$. Using these normal fibres we can define the family $\mathscr{F}$ of $2 n$ fibres that will have the property $\mathscr{F}=V \cap\left(D_{1} \cup \cdots \cup D_{n}\right)$. In every annulus from $V-V_{1}$ the family $\mathscr{F}$ consists of a single copy of the fibre $\lambda$. In the annulus $V_{1}$ we take $2 g+1-\chi(B)$ (resp. $g+1-\chi(B)$ ) copies of $\lambda$ if the base surface $B$ is orientable (resp. nonorientable). We will now give a recursive definition of the collection of vertical compression discs (compare [Mijatović 2004, Section 6.2]). Assuming that we have already created a sub-collection $D_{1}, \ldots, D_{k}$, for some $k<n$, whose vertical boundary lies in $\mathscr{F}_{F}$ and which satisfies all other requirements, we look at the $I$-bundle $M_{k}=M-\operatorname{int} \mathcal{N}\left(D_{1} \cup \cdots \cup D_{k}\right)$ which inherits a natural polyhedral structure from the original triangulation of $M$. Any choice of the vertical disc $D_{k+1}$ has to lie in $M_{k}$ and can be made normal with respect to this polyhedral decomposition. It is precisely defined as follows.

Proposition 4.8. Let $M \rightarrow B$ be a triangulated I-bundle over a (possibly nonorientable) bounded surface $B$ and let $D_{1}, \ldots, D_{k}$, where $k \in\{1, \ldots, n-1\}$, be normal vertical compression discs as described above. Fix two normal arcs $e$ and $f$ from $\mathscr{F} \cap M_{k}$, which are not contained in the union $D_{1} \cup \cdots \cup D_{k}$ and are supposed to be the vertical boundary of the next disc in our collection. Let $D_{k+1}$ be the normal vertical compression disc whose vertical boundary consists of $e \cup f$ and which minimises the weight, with respect to the polyhedral structure on 
$M_{k}$, among all normal vertical compression discs that carry nontrivial elements of $H_{2}\left(M_{k}, \partial M_{k} ; \mathbb{Z}_{2}\right)$ and are bounded by $e \cup f$. Then $D_{k+1}$ is fundamental in $M_{k}$.

Proposition 4.8 is very similar to Proposition 6.3 of [Mijatović 2004]. Its proof is simpler because the incompressible summands we need to deal with are never horizontal. Since the triangulation of $M$ induces a simplicial structure on the vertical boundary $V$, we can use $\partial V$ as a boundary pattern on $M$ (or even on $M_{k}$ ). This enables us to apply our usual techniques. The proof of Proposition 4.8 is by contradiction. We assume that our disc $D_{k+1}$ is a sum of two normal surfaces. Then, using the familiar patch arguments, we can show that one of the summands has to be a vertical disc and the other one is a pure vertical annulus. To get a contradiction we then proceed in exactly the same way as in the proof of Proposition 6.3 in [Mijatović 2004]. The details are left for the reader.

To make sure that, after the first compression along $D_{1}$, the base surface of the $I$-bundle $M_{1}$ is orientable, we proceed exactly as in [Mijatović 2004, Section 6.2]. Also the same bounds on the normal complexity apply in the setting of Proposition 4.8. Hence our chosen family of compression discs contains no more than

$$
\left(2 \cdot 2^{11 t}\right)^{12 t}<2^{80 t^{2}}
$$

discs (for the proof see the discussion in [Mijatović 2004], just before Lemma 6.4). Since any automorphism of $B$, that is fixed on the boundary, extends to a homeomorphism of the $I$-bundle $M$, we can use Lemma 6.4 in [Mijatović 2004] to go between any two such families of compression discs.

\section{Proof of the Main Theorem}

Now we can prove Theorem 3.1. What we need to do is to connect any two triangulations of a given fibre-free Haken 3-manifold using Pachner moves. First we subdivide both triangulations so that the characteristic submanifold $\Sigma$ is triangulated by a subcomplex in each of the subdivisions. In the strongly simple pieces of the JSJ-decomposition which are not contained in $\Sigma$, the subdivisions are then further simplified using the distinguished hierarchy. The gap between the triangulations in the components of $\Sigma$ is bridged by Theorem 3.1 of [Mijatović 2004] if they are Seifert fibred and by Proposition 4.8 if they are $I$-bundles.

Before continuing with the proof we should note that Theorem 3.1 is not necessarily false for Haken 3-manifolds which are not fibre-free. The only reason for the fibre-free hypothesis is to make sure that we can construct the distinguished hierarchy in all strongly simple pieces of the JSJ-decomposition of our 3-manifold $M$. If, for example, our manifold is a surface bundle over the circle, then, after cutting along the fibre, there are no distinguished surfaces in the cut-open manifold (which is an $I$-bundle) to continue the hierarchy. One possible way around this 
problem in the surface bundle case, is to look carefully for the first surface, making sure that it is not a fibre. Such a strategy however could not work for all surface bundles because a nonfibre does not always exist and even when it does, it is not clear how to construct it algorithmically.

Going back to the proof of Theorem 3.1, let $M$ be a fibre-free Haken 3-manifold with a triangulation $T$ that contains $t$ tetrahedra. We are now going to construct a distinguished triangulation $\bar{T}$ of the complement of the characteristic submanifold $\Sigma$. We start by subdividing $T$ so that the distinguished hierarchy appears as a subcomplex of this subdivision. This subcomplex induces a simplicial structure of the manifold $M-$ int $\Sigma$ which is uniquely determined by the topology of $M$. The new triangulation will be closely related to the distinguished hierarchy which was described in Section 4. We know that each complementary piece of the distinguished hierarchy in $M-$ int $\Sigma$ is a 3-ball or a solid torus with at least one pure annulus in its boundary. So we can define $\bar{T}$ to be conical in each of the 3-balls. Let $K$ be the two-dimensional polyhedron which is a union of surfaces in the distinguished hierarchy. In order to avoid confusion we should emphasise that $K$ also contains the surfaces from $\partial(M-$ int $\Sigma)$. The complement of the singular locus of $K$ is a disjoint union of discs and pure annuli which live in the boundaries of the solid tori. The discs will be contained in the 2-skeleton of $\bar{T}$ and will be triangulated as cones on their boundaries. Since the singular locus of $K$ is a graph which is embedded in the 3-manifold $M$, it already has a canonical simplicial structure. This induces a triangulation on each of the boundaries of the two-dimensional faces of the polyhedron $K$. So by definition the triangulation $\bar{T}$ is uniquely determined by the distinguished hierarchy in the complementary regions which are 3-balls.

We still need to define $\bar{T}$ in the complementary pieces which are solid tori with pure annuli in their boundaries. Notice that it follows directly from the definition of the boundary pattern that a pure annulus in the boundary of a complementary solid torus cannot be homotopically trivial. It therefore induces a unique Seifert fibration of the whole piece. If there are several pure annuli in the boundary of a single solid torus then they must all be disjoint. So they induce the same Seifert fibration of the piece. Therefore the union of all such solid tori is a disjoint union of Seifert fibred spaces. Furthermore there is a simplicial structure on all boundary components of this Seifert fibred space which is induced by the singular locus of $K$. So we can take $\bar{T}$ to be the simplified triangulation of our Seifert fibred space that was defined in the proof of Theorem 3.1 in [Mijatović 2004].

Now we need to construct $\bar{T}$ using Pachner moves. The starting point is the original triangulation $T$ of $M$. Our main tool for subdividing a triangulation, so that the subdivision contains a given normal surface in its 2-skeleton, will be Lemma 4.1 from [Mijatović 2004]. Before we start estimating the number of Pachner moves 
we need to make, we should remind ourselves that the notation $E^{n}(x)$ stands for the composition of the exponential function $E(x)=2^{x}$ with itself $n$ times. By Lemma 4.6 the surface $S_{1}$ consists of $2^{350 t^{2}}$ normal discs in the triangulation $T$. Making less than $200 t \cdot 2^{350 t^{2}}<2^{360 t^{2}}$ Pachner moves we can subdivide $T$ so that the subdivision contains $S_{1}$ in its 2 -skeleton. The number of 3 -simplices in the subdivision is bounded above by $s=20\left(t+2^{350 t^{2}}\right)<2^{360 t^{2}}$. We also know (see the discussion after Lemma 4.6) that the surface $S_{2}$ consists of not more than $20 t \cdot 2^{80 s^{2}}<2^{90 s^{2}}$ normal discs in the subdivision. Applying Lemma 4.1 of [Mijatović 2004] again, we see that

$$
200 \cdot 2^{90 s^{2}} 2^{360 t^{2}}<2^{100 s^{2}}<E^{2}\left(730 t^{2}\right)
$$

bounds the number of Pachner moves needed to construct the subdivision of $T$ which supports $S_{1} \cup S_{2}$ as a subcomplex. The same expression bounds the number of tetrahedra in this subdivision.

In order to see how much more we need to subdivide the current triangulation of $M-$ int $\Sigma$ if we want it to contain the polyhedron $K$ in its 2-skeleton, we have to estimate the number of connected surfaces that arise during the implementation of Step 3. Corollary 4.5 implies that the sum of the topological complexities of all closed surfaces in $M$, which bound complementary pieces after the first two steps of the hierarchy, is bounded above by $2^{150 t}$. The $P$-canonical annuli are never $\partial$-parallel in the piece they appear in. The same is true of the spanning annuli which feature in $3 \mathrm{~b}$. We have already established, when we were proving that the distinguished hierarchy has to terminate, that there can be at most $9 g(\partial H)$ disjoint nonparallel incompressible annuli which are not boundary parallel in any complementary piece $H$.

It is clear from the construction that all $P$-canonical annuli that occur in a single complementary piece $H$ are disjoint and therefore not parallel (the annuli we add after we have cut $H$ for the first time are vertical in the product structure given by Theorem 2.1). So $2 \cdot 9 \cdot 2^{150 t}$ is an upper bound on the number of such annuli in the distinguished hierarchy (the factor 2 is there because we sometimes need to add two parallel copies of a surface). The following expression

$$
40 t+\left(2-\chi\left(S_{2}\right)\right)+18 \cdot 2^{150 t}<20 \cdot 2^{150 t}
$$

bounds the number of $P$-essential annuli in the distinguished hierarchy. We have shown (see page 168) that the first two summands control the number of boundary components of $S_{2}$ and hence the number of trivial $P$-essential annuli in $K$. The number of $P$-canonical annuli is controlled by the exponential expression at the end. The inequality follows from the bound on $2-\chi\left(S_{2}\right)$ which can be found in the proof of Corollary 4.5. We can now conclude that the total number of surfaces in Step 3 of the hierarchy is bounded above by $2^{160 t}$. 
Proposition 2.5 and Lemma 4.7 tell us that if we are looking for any of the surfaces in some complementary piece with $r$ tetrahedra, we can construct it by using less than $2^{80 r^{2}}$ normal discs. By Lemma 4.1 in [Mijatović 2004] we need to make not more than $200 r 2^{80 r^{2}}$ Pachner moves to make this surface part of the 2 -skeleton of the subdivision. The same expression also bounds the number of tetrahedra in the subdivision. It is clearly smaller than $E^{2}(r)$ for $r$ larger than say 100. Since the numbers we are going to apply this to are significantly bigger than that, we can use this bound. In other words we can make the whole polyhedron $K$ a subcomplex of some subdivision of the triangulation of $M$ by making less than

$$
E^{2 \cdot 2^{160 t}}\left(E^{2}\left(730 t^{2}\right)\right)<E^{2 \cdot 2^{160 t}}\left(E^{3}(10 t)\right)<E^{2^{170 t}}(t)
$$

moves. Again this expression bounds the number of 3-simplices involved. Now we have to apply Theorem 5.2 in [Mijatović 2004] to every complementary 3-ball region of $K$ in order to make it conical. Theorem 3.1 from [Mijatović 2004] can be used to deal with Seifert fibred spaces which are unions of solid tori that had pure annuli in their boundaries. Since $r$ (i.e. the number of tetrahedra) at this stage is so large, the bounds in those theorems are certainly smaller than $E^{7}(r)$. The number of 3-balls and Seifert fibred pieces we need to deal with is bounded above by the number of 3 -simplices in the subdivision. Also the procedures simplifying the simplicial structure of the faces of $K$ are linear in the number of tetrahedra of the subdivision. The amalgamation of the edges in the singular locus of $K$ takes linearly many steps as well. Processes very similar to these are described in the proof of Theorem 6.5 in [Mijatović 2004]. So we can assume that after $E^{2^{180 t}}(t)$ Pachner moves our subdivision looks like $\bar{T}$.

Proposition 4.8 implies that a triangulation of an $I$-bundle over a bounded surfaces can be dealt with in the same way Theorem 6.5 in [Mijatović 2004] deals with $S^{1}$-bundles over bounded surfaces. In fact we can obtain a complete analogue of Theorem 6.5 from [Mijatović 2004] for $I$-bundles over bounded surfaces. We then apply it to the $I$-bundle components of $\Sigma$. All that is left now is to apply Theorem 3.1 of [Mijatović 2004] to the Seifert fibred components of $\Sigma$. This gives the bound from Theorem 3.1. If our manifold is an $I$-bundle over a closed surface which is not Seifert fibred, we first look for some vertical annulus which is fundamental, and then do the procedure described above to its complement. Clearly the bound from Theorem 3.1 still applies.

\section{Acknowledgments}

We thank the referee for many constructive comments and suggestions that improved the paper immensely. 


\title{
References
}

[Bart and Scharlemann 1996] A. Bart and M. Scharlemann, "Least weight injective surfaces are fundamental”, Topology Appl. 69:3 (1996), 251-264. MR 97c:57012 Zbl 0858.57016

[Gordon and Luecke 1989] C. M. Gordon and J. Luecke, "Knots are determined by their complements", J. Amer. Math. Soc. 2:2 (1989), 371-415. MR 90a:57006a Zbl 0678.57005

[Hass and Lagarias 2001] J. Hass and J. C. Lagarias, "The number of Reidemeister moves needed for unknotting”, J. Amer. Math. Soc. 14:2 (2001), 399-428. MR 2001m:57012 Zbl 0964.57005

[Hass et al. 1999] J. Hass, J. C. Lagarias, and N. Pippenger, "The computational complexity of knot and link problems", J. ACM 46:2 (1999), 185-211. MR 2000g:68056

[Hempel 1976] J. Hempel, 3-Manifolds, Annals of Mathematics Studies 86, Princeton University Press, Princeton, 1976. MR 54 \#3702 Zbl 0345.57001

[Jaco 1980] W. Jaco, Lectures on three-manifold topology, CBMS Regional Conf. Series in Math. 43, American Mathematical Society, Providence, 1980. MR 81k:57009 Zbl 0433.57001

[Jaco and Shalen 1979] W. H. Jaco and P. B. Shalen, Seifert fibered spaces in 3-manifolds, Mem. Amer. Math. Soc. 220, Amer. Math. Soc., Providence, 1979. MR 81c:57010 Zbl 0415.57005

[Johannson 1979] K. Johannson, Homotopy equivalences of 3-manifolds with boundaries, Lecture Notes in Mathematics 761, Springer, Berlin, 1979. MR 82c:57005 Zbl 0412.57007

[Lackenby 2003] M. Lackenby, "Exceptional surgery curves in triangulated 3-manifolds", Pacific J. Math. 210:1 (2003), 101-163. MR 2004d:57024 Zbl 02101082

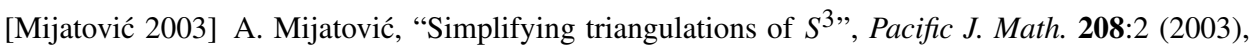
291-324. MR 2004f:52014

[Mijatović 2004] A. Mijatović, “Triangulations of Seifert fibred manifolds”, Math. Ann. 330:2 (2004), 235-273. MR 2089425

[Neumann and Swarup 1997] W. D. Neumann and G. A. Swarup, "Canonical decompositions of 3-manifolds", Geom. Topol. 1 (1997), 21-40. MR 98k:57033 Zbl 0886.57009

[Pachner 1991] U. Pachner, "P.L. homeomorphic manifolds are equivalent by elementary shellings", European J. Combin. 12:2 (1991), 129-145. MR 92d:52040 Zbl 0729.52003

[Thurston 1986] W. P. Thurston, “A norm for the homology of 3-manifolds", pp. i-vi and 99-130 in Mem. Amer. Math. Soc. 339, American Mathematical Society, Providence, 1986. MR 88h:57014 Zbl 0585.57006

[Tollefson and Wang 1996] J. L. Tollefson and N. Wang, "Taut normal surfaces", Topology 35:1 (1996), 55-75. MR 97j:57022 Zbl 0868.57022

[Waldhausen 1968] F. Waldhausen, "On irreducible 3-manifolds which are sufficiently large", Ann. of Math. (2) 87 (1968), 56-88. MR 36 \#7146 Zbl 0157.30603

Received April 19, 2003. Revised May 29, 2004.

\author{
ALEKSANDAR MiJatović \\ Department of Pure Mathematics and Mathematical Statistics \\ CENTER For MATHEMATICAL SCIENCES \\ UNIVERSITY OF CAMBRIDGE \\ WILBERFORCE ROAD \\ CAMBRIDGE, CB3 0WB \\ UNITED KINGDOM \\ a.mijatovic@dpmms.cam.ac.uk
}

